\title{
EXPERIMENTAL DRUG TREATMENT OF EAST COAST FEVER OF CATTLE.
}

\author{
By GEORGE H. F. NUTTALL, F.R.S. \\ (From the Quick Laboratory, University of Cambridge.) \\ (With 1 Chart.)
}

THE results obtained in the treatment of East Coast Fever have been uniformly negative. Nevertheless it appears desirable to publish the records of 18 experimental cases which we have treated with various drugs, since the negative evidence afforded may help other workers in the search for the remedy which we must hope will ultimately be found.

Trypanblue was found to be ineffective by Nuttall and Hadwen (v. 1909, Parasitology, II. p. 190), in one experiment the dye "exerted no effect whatsoever on the parasite." The record and chart of the case accompanies the paper by Nuttall, Fantham and Porter (9. II. 1910, Parasitology, II. pp. 329, 331, 332). A cow was infected by means of Rhipicephalus evertsi adults, she showed fever on the 14th day, and a few parasites were found in her blood 20 days after the ticks had been placed upon the animal. The trypanblue was given intravenously on the 21 st (200 c.c. of $1 \%$ solution), 22 nd ( 200 c.c. of $2 \%$ solution) and 23 rd day ( 100 c.c. of $2 \%$ solution), but the number of parasites steadily increased until the animal died. Negative results were subsequently obtained in a field trial conducted in South Africa. We have shown that trypanblue is an excellent remedy for piroplasmosis in dogs and cattle, and it has been found effective in biliary fever in horses. As the question is frequently asked "What is trypanblue?" I herewith append the formula $\left(\mathrm{C}_{34} \mathrm{H}_{24} \mathrm{~N}_{6} \mathrm{O}_{14} \mathrm{~S}_{4} \mathrm{Na}_{4}\right.$; the tetrazo compound of toluidine and amidonaphtholsulphonate of sodium).

The drugs used in the experiments herein recorded were the following:

I. Congo red ("concentrated," obtained from Messrs Levinstein, Ltd., Blackley, near Manchester). It was used once, 70 o.c. of a 
$0.5 \%$ solution being injected subcutaneously into Calf 8 (see protocol of Exp. I) before the parasites appeared in the animal's blood, Koch's "blue bodies" having, however, been found in gland smears coincidently with the onset of fever. The administration of this dye did not retard the appearance of the parasites in the blood. The dye was tried because it had been found by me to exert an effect on Piroplasma canis (Parasitology, II. p. 415).

I a. Sodium salicylate $(10 \%)+$ Sodium bicarbonate $(1 \%)$ in 100 c.c. of $\mathrm{NaCl}$ solution was subsequently injected intravenously into Calf 8 on three days, beginning on the second day after the appearance of the parasites in the blood, but the result was negative.

II-III. Tryposafrol (according to Prof. Brieger and Dr Krause, kindly supplied by the Aktien-Gesellschaft für Anilin Fabrikation, Berlin). This dye was given to two calves. In Exp. II it was given in daily doses ranging from 0.25 to 3 grammes dissolved in sugared water which the animal drank. Treatment began on the fifth day after the onset of fever and for six days before the parasites appeared in the animal's blood, and continued daily up to and including the day of death. The number of parasites in the blood remained small but they showed a definite increase in spite of treatment. In Exp. III the calf received the same treatment, the dose of tryposafrol being 0.25 , rising to 1.5 grammes daily; treatment began on the day after the first parasites appeared in the animal's blood and was maintained to the end. The number of parasitized corpuscles steadily increased in spite of treatment. (See protocol on p. 63.) This dye was tried because of the claims made by Brieger and Krause that it exerted a curative effect in piroplasmosis of cattle and in trypanosomiasis. Our experiments with tryposafrol in relation to piroplasmosis and trypanosomiasis will be reported upon in a future paper.

IV. Creosote 0.2 c.c. + Oleum copaivae 3.7 c.c. in $3 \%$ solution injected intravenously in seven daily doses of 5 c.c. beginning on the third day of fever and three days before the appearance of parasites in the animal's blood. No effect was exerted by the treatment (see protocol, p. 64). Creosote was chosen as a representative of antiseptic drugs belonging to the volatile aromatic series, being more powerfully antiseptic than carbolic acid and possessing greater penetrating power. The copaiva was added as a representative of the less irritant volatile oils with antiseptic properties. 


\section{8 . Drug Treatment of East Coast Fever of Cattle}

V. Arsacetin (sodium-p-acetyl-aminophenylarsinate-acetylarsanilate. Obtained from Farbwerke vorm. Meister Lucius und Brüning, Hoechst a. M.) was given in doses of 2 to 5 c.c. of a $5 \%$ solution, intravenously on seven alternate days. Treatment began on the day after the parasites appeared in the animal's blood. The drug exerted no effect, as will be seen by reference to the protocol on p. 65 .

VI-VII. Soamin (sodium-para-amino-phenylarsonate. Obtained from Messrs Burroughs Wellcome and Co.). In Exp. VI 2.5 grammes dissolved in 12.5 c.c. of hot water and injected intramuscularly, whilst warm, on seven days. Treatment began on the day when parasites first appeared in the calf's blood. In Exp. VII a $2 \%$ solution was injected intravenously in doses ranging from 3 to 13 c.c. following on the injection of 2 to 4 c.c. of a solution of eosin and sodium bicarbonate ( $\frac{1}{2}$ a grain of each per c.c.). The treatment began on the fifth day after the parasites appeared in the calf's blood. In both experiments the drug exerted no influence on the progress of the disease. (See protocols, pp. 66, 67.)

VIII. " 606 " : a single dose of 2 grammes was injected intravenously on the third day after the parasites appeared in the cow's blood. The drug did not produce any visible effect on the parasites and the animal died of East Coast Fever within 48 hours. (See protocol, p. 68.)

IX. Emetine hydrochloride, $1 \%$ in $\mathrm{NaCl}$ solution was injected intravenously in doses ranging from 1 to 10 c.c. on nine successive days, beginning on the second day of the appearance of parasites in the calf's blood. The drug did not affect the course of the disease, the number of parasitized corpuscles in the peripheral blood mounted steadily until the animal died. (See protocol, p. 69.) The drug was used because of the favourable reports relating to its curative action in Amoebiasis.

X. Mercury salicylate, $1 \%$ in $0.8 \% \mathrm{NaCl}$ solution was injected intravenously in doses ranging from 4 to 20 c.c. on nine days. Treatment was commenced two days before the parasites appeared in the animal's blood. The drug exerted no retarding effect upon the progress of the disease. (See protocol, p. 70.)

XI. Mercury succinimide $\left(\mathrm{Hg}\left[\mathrm{C}_{2} \mathrm{H}_{4}\left(\mathrm{CO}_{2}\right) \mathrm{N}\right]_{2}\right)$ in $3 \%$ solution was injected intravenously in three doses of 2,4 and 3 c.c. in as many days, beginning on the day when the first parasites appeared in minimal 
numbers in the calf's blood. The drug exerted no effect whatever. (See protocol, p. 71.)

XII-XIII. Quinine bihydrochloride. In Exp. XII the animal received on 14 successive days subcutaneous injections of 55 to 60 grains dissolved in 25 c.c. of water. Treatment commenced on the third day of the appearance of parasites in the animal's blood. In Exp. XIII quinine hydrochloride was given on five successive days followed by the bihydrochloride on seven out of nine days, the drug being injected intramuscularly. Treatment in this case began on the first appearance of parasites in the calf's blood. No effect was observable from the treatment. (See protocols, pp. 72-74.)

XIV. Ethylhydrocupreine $\mathrm{HCl}, 24$ c.c. of an $8 \%$ solution was injected subcutaneously on five successive days, beginning on the second day after the appearance of parasites in the calf's blood. The result was negative. (See protocol, p. 75.) This drug had been recommended because of its bactericidal effect in pneumococcus infections.

$\mathrm{XV}$. Ammonium fluoride, $0.5 \%$ in $0.8 \% \mathrm{NaCl}$ solution was injected in doses of 18 to 20 c.c. intravenously on nine successive days. Treatment began on the second day of the parasites appearing in the calf's blood. There was no observable effect. (See protocol, p. 76.) The trial of ammonium fluoride was suggested by its known inhibitive effect on bacteria producing lactic and butyric acid fermentation without interfering with the growth of yeast.

XVI. Potassium iodide in $25 \%$ solution was injected intravenously on five successive days, the dose ranging from 20 to 40 c.c. Treatment commenced a day before the parasites appeared in the animal's blood but the result was negative. (See protocol, p. 77.)

XVII. Calcium lactate in $10 \%$ solution was injected intravenously on seven successive days, the dose ranging from 3 to 5 c.c. Treatment began on the second day of the appearance of parasites in the calf's blood. There was no effect observable. (See protocol, p. 78.)

XVIII. Nuclein from thymus gland, 10 c.c. of a $5 \%$ solution was given subcutaneously on five days, commencing on the third day of fever and two days before the parasites appeared in the calf's blood. The treatment was without effect. (See protocol, p. 79.) 


\section{Drug Treatment of East Coast Fever of Cattle}

\section{Records of Cattle subjected to Drug Treatment.}

When the experiments took place in winter the animals were kept in stalls warmed to $60-70^{\circ} \mathrm{C}$. The ticks used for infecting the animals were enclosed in bags fastened about the ears of the cattle.

Blood films were prepared from the animals every day from the time that fever occurred. In some cases films were prepared twice a day or oftener. The films were stained by Giemsa's method. To determine the percentage of infected red blood corpuscles it was usually found sufficient to count 250 corpuscles all told (infected and uninfected), but on some occasions 500 to 1000 were counted so as to arrive at a more exact result.

Autopsies were made and the presence of typical lesions was noted in all cases.

\section{Experiment I (Calf 8).}

Treatment: (1) Congo red, $0.5 \%$ solution, 70 c.c. injected subcutaneously on the 14th day.

(2) Sodium salicylate, $10 \%$ solution + Sodium bicarbonate, $1 \%$ in normal $\mathrm{NaCl}$ solution, 100 c.c. injected intravenously on the 21st, 22nd and 24th day.

(3) Mercury salicylate in $1 \%$ solution, 5 c.c. injected intravenously on the 29th day (too late).

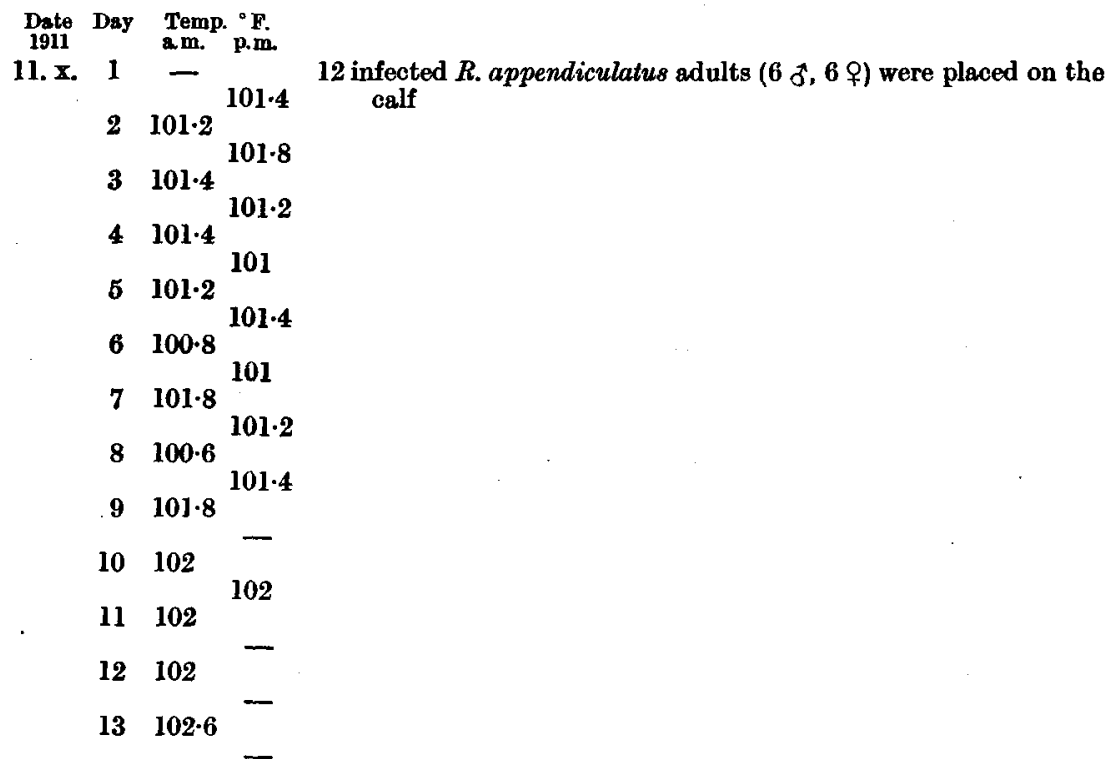




\section{EXPERIMENT I (cont.).}

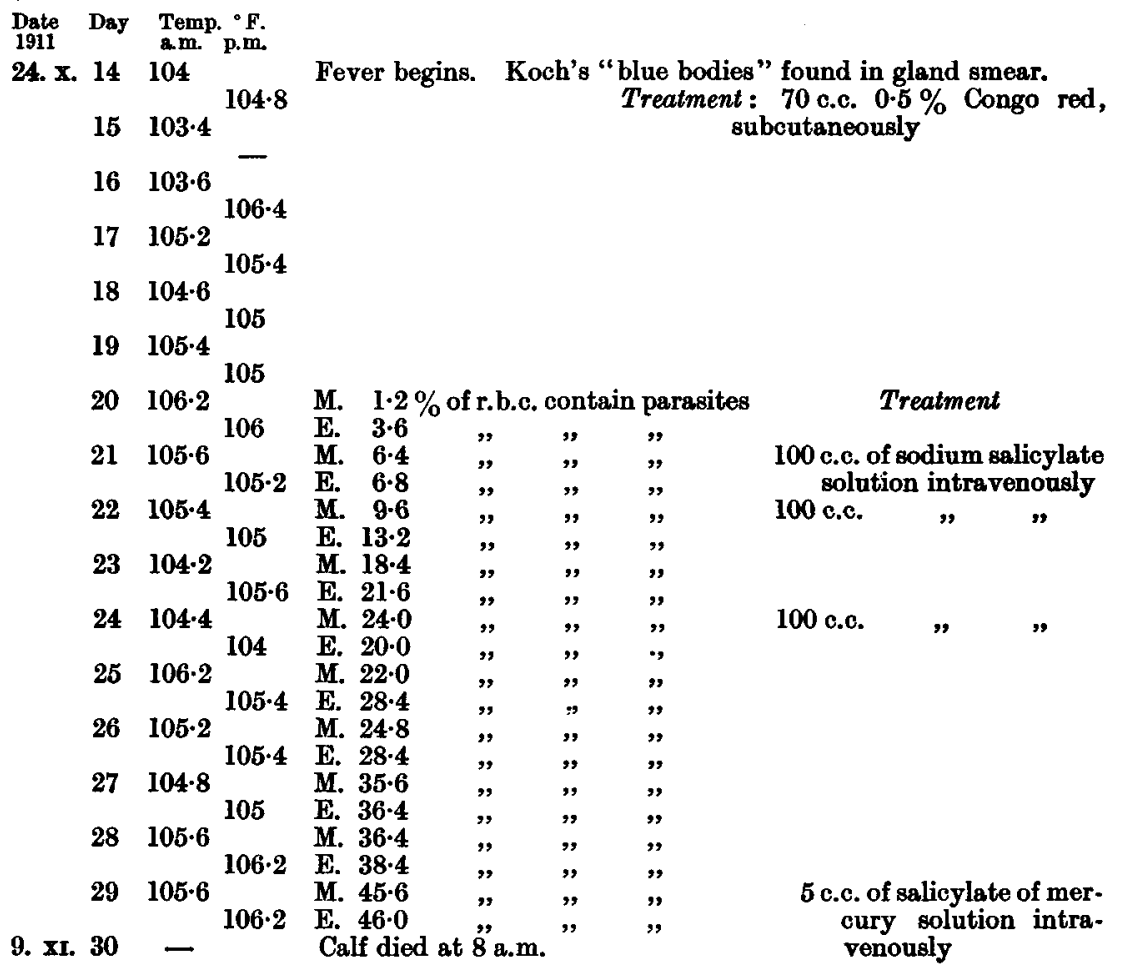

\section{Experiment II (Calf 18).}

Treatment: Tryposafrol, $0 \cdot 25$ grammes rising to 3 grammes daily in 5 litres of sugared water on 17 successive days.

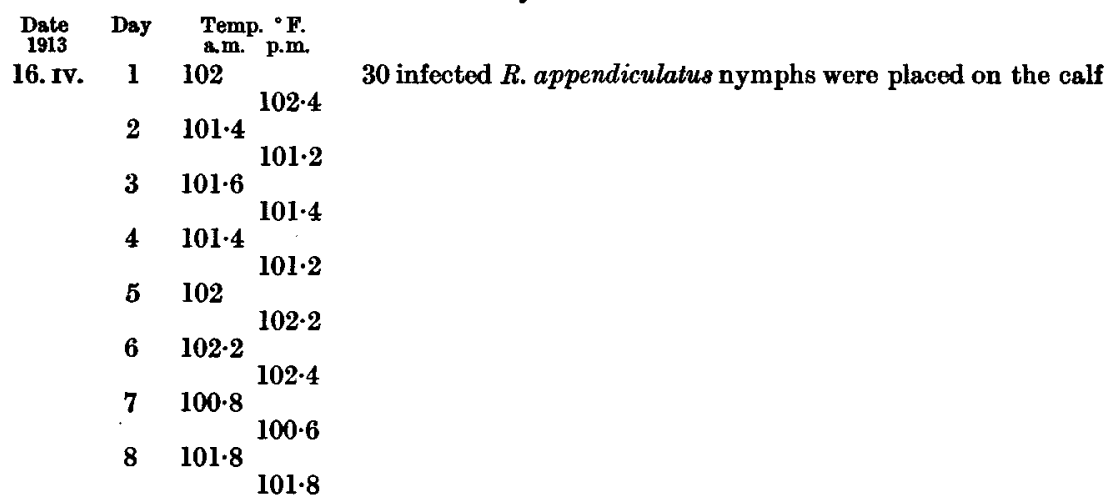


62 Drug Treatment of East Coast Fever of Cattle

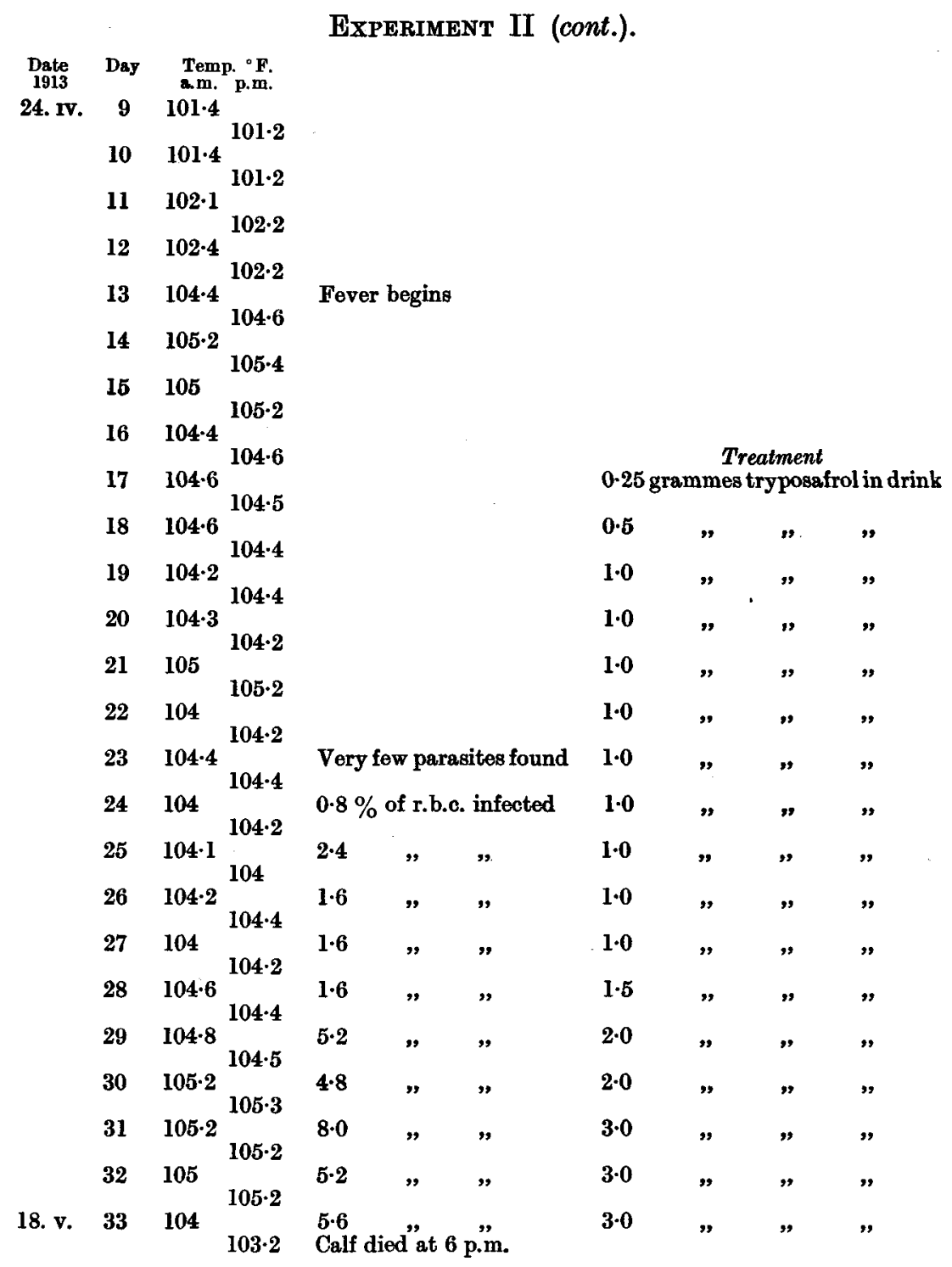




\section{Experiment III (Calf 19).}

Treatment: Tryposafrol, 0.25 rising to 1.5 grammes daily in 5 litres of sugared water as a drink on 11 successive days.

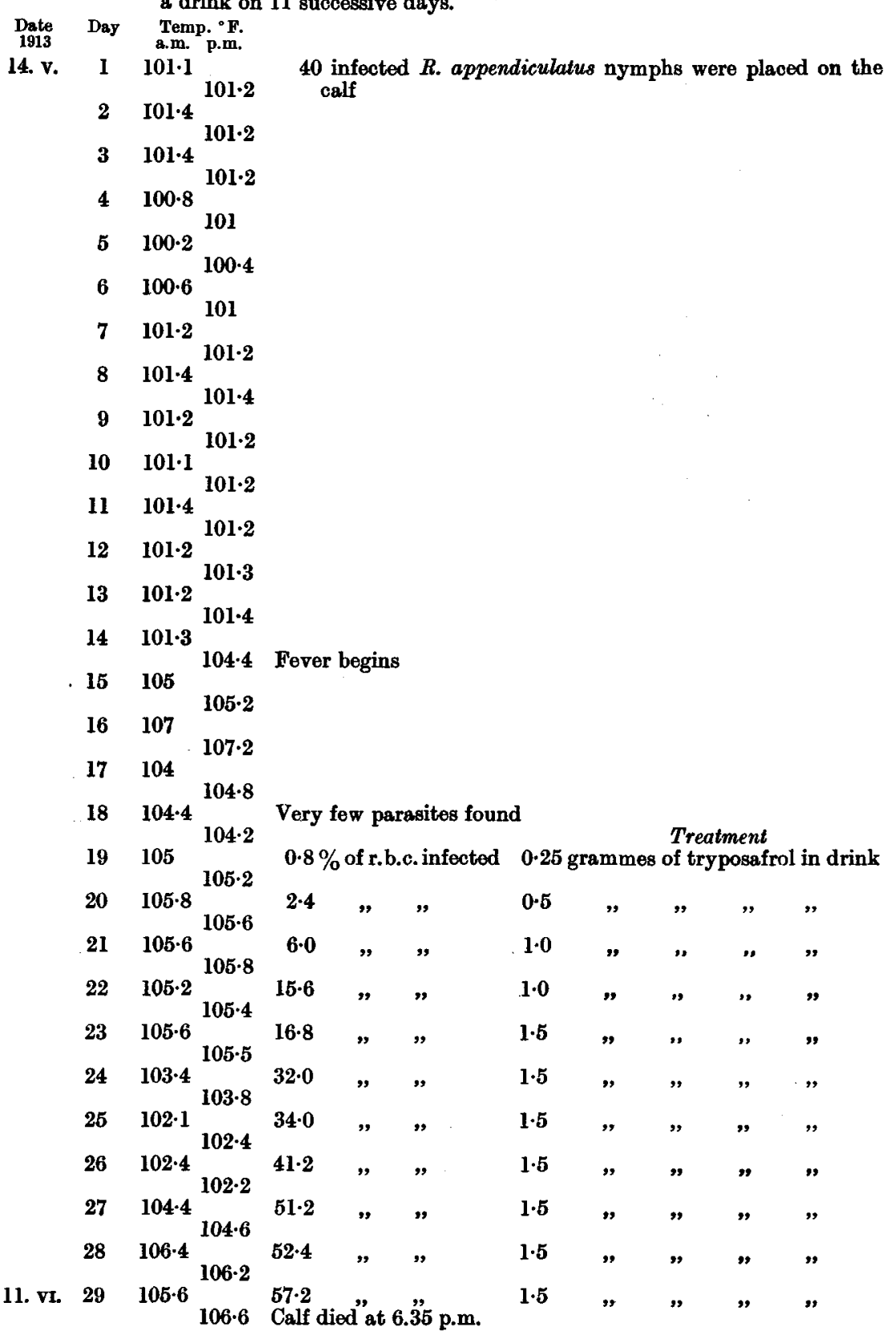




\section{Drug Treatment of East Coast Fever of Cattle}

\section{ExPERTMENT IV (Calf 7).}

Treatment: Creosote and Oleum copaivae (Creosote 0.2 c.c. + Ol. copaivae 3.7 c.c. diluted to form a $3 \%$ solution).

Date Day Temp. ${ }^{\circ} \mathrm{F}$.

29. I1. 1 101.2 12 infected $R$. appendiculatus adults (6 $\%, 6$ o) were placed on 101.6 the calf

$101 \cdot 2$

$3 \quad 101 \cdot 2$

$4 \quad 101 \cdot 2 \frac{101 \cdot 4}{101}$

$5 \quad 101 \cdot 6$

$6100 \cdot 8$

$7 \quad 101 \quad 101.4$

$8 \quad 101.6^{101 \cdot 2}$

$\begin{array}{lll}8 & 101 \cdot 6 & 101 \cdot 3\end{array}$

$\begin{array}{lll}10 & 102 & 101\end{array}$

$11 \quad 101^{101 \cdot 8}$

$12 \quad 102 \cdot 8$

$13 \quad 104 \cdot 2{ }_{103 \cdot 2}^{102.4}$ Fever begins

$14 \quad 105 \cdot 2$

$15 \quad 106^{105 \cdot 6}$

$16 \quad 105 \cdot 4$

$106 \cdot 2$

$17 \quad 106 \cdot 2$

$18 \quad 105 \cdot 8$

$19 \quad 105 \cdot 4$

$20105 \quad 105$

$05 \cdot 6$

Very few parasites found

5 c.c. of drug injected intravenously 5 c.c.

5 c.c. , , , ,

5 c.c. , , ,

$\begin{array}{lll}21 & 104 \cdot 8 & \\ & & 104 \cdot 8\end{array}$

$\begin{array}{lll}22 & 105 & 104 \cdot 6\end{array}$

$23 \quad 104 \cdot 22^{104 \cdot 6} 16$.

$24104 \cdot 6^{104 \cdot 4} 20$

$\begin{array}{llll}25 & 105 & 104 \cdot 8 & 31\end{array}$

$26 \quad \begin{array}{llll}105 \cdot 8 & 105 \cdot 2 & 31 \cdot 6 \\ & & 39 \cdot 6\end{array}$

$27 \quad 106.8$

$28 \quad 106 \quad 106 \cdot 2$

$105 \cdot 6$

$97 \cdot 6$ 
EXPERIMENT V (Calf 6).

Treatment: Arsacelin, $5 \%$ solution given on 7 alternate days in doses ranging from 2 to 5 c.c. intravenously.

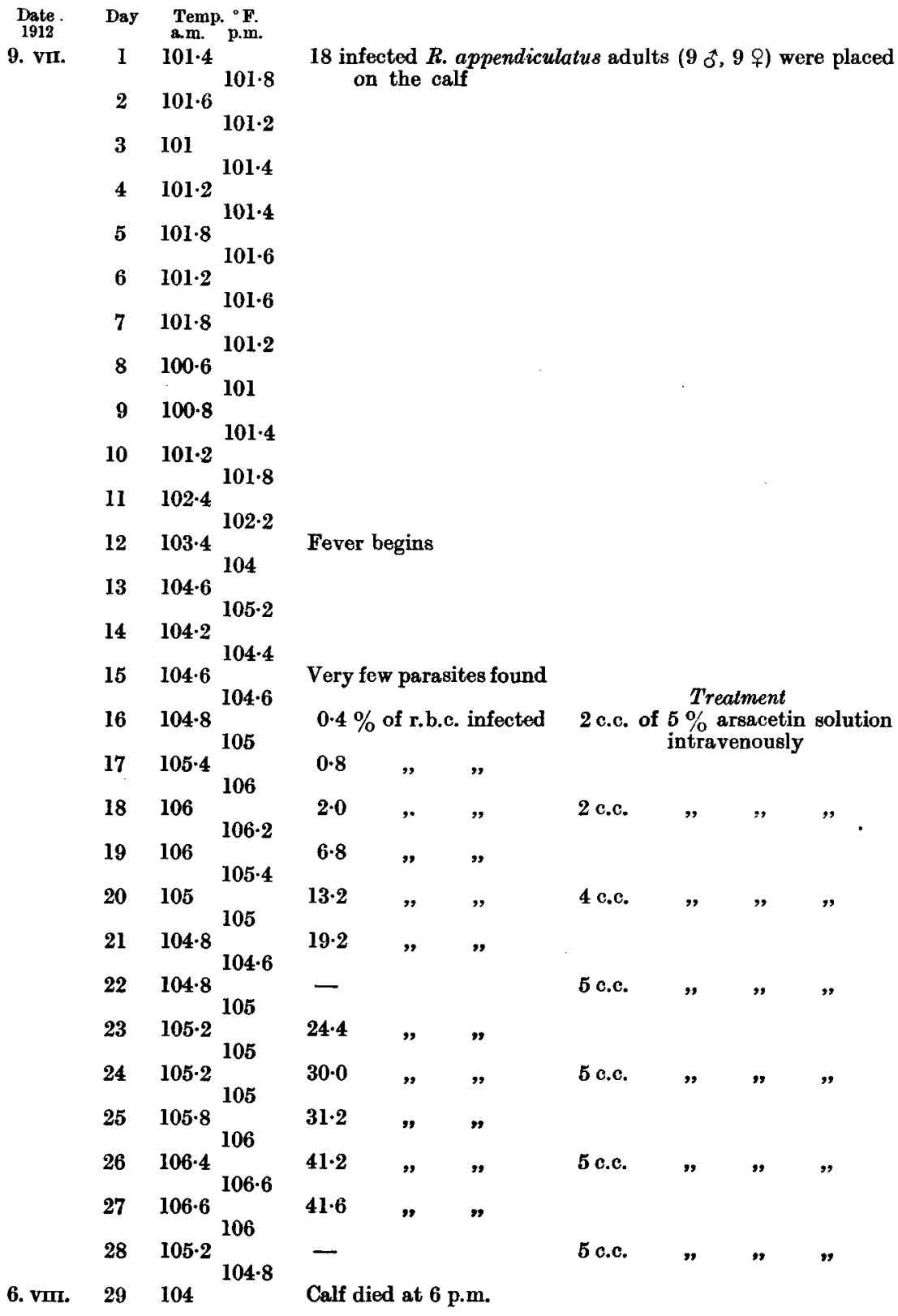




\section{Drug Treatment of East Coast Fever of Cattle}

\section{EXPERIMENT VI (Calf 3).}

Treatment: Soamin, 2.5 grammes dissolved in 12.5 c.c. of hot water and injected intramuscularly, whilst warm, on 7 days.

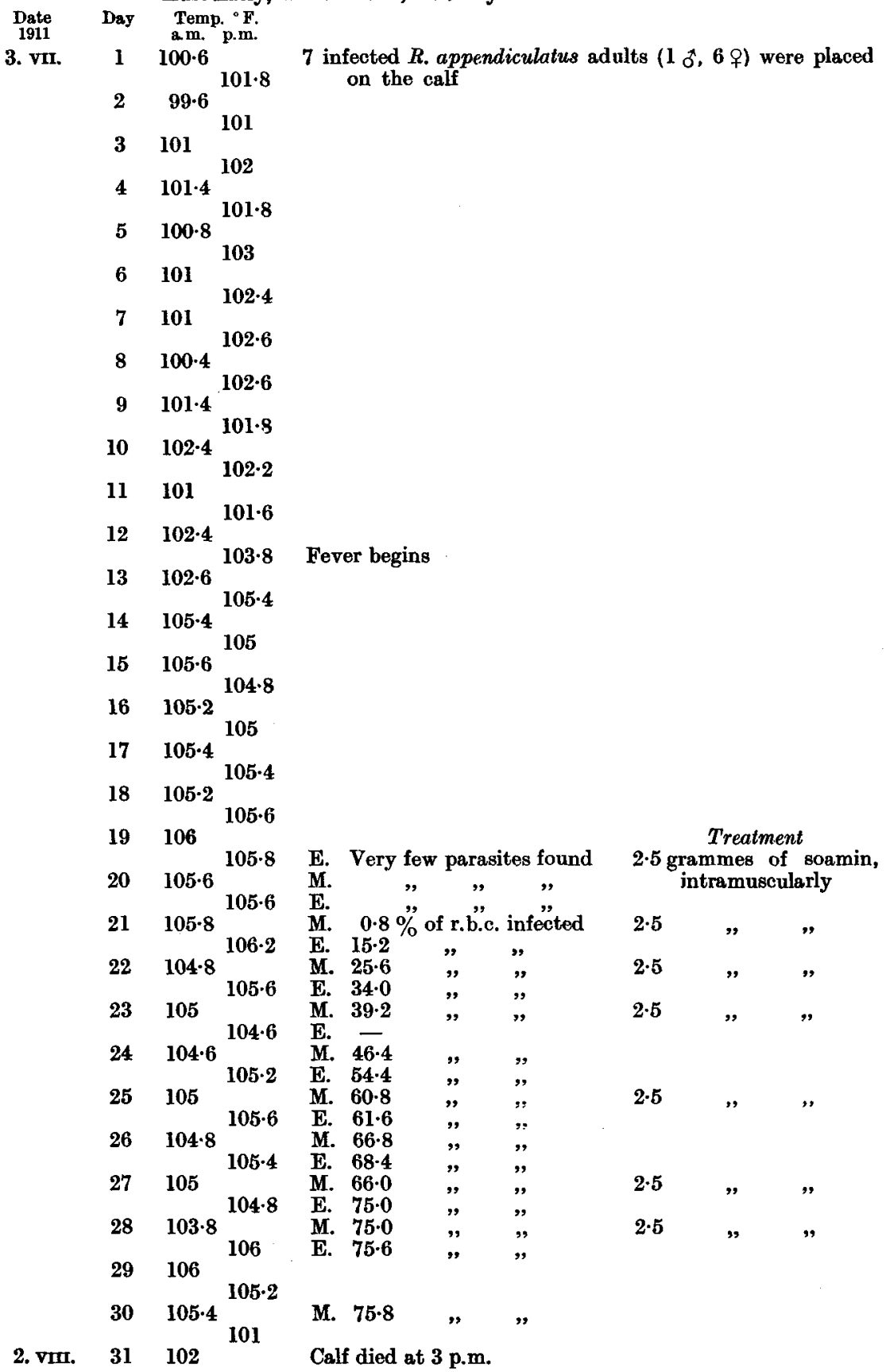


EXPERIMENT VII (Calf 11).

Treatment: Soamin, $2 \%$ solution given intravenously in doses ranging from 3 to 13 c.c., following on the intravenous injection of 2 to 4 c.c. of a solution of eosin and sodium bicarbonate ( $\frac{1}{2}$ a grain of each per c.c.).

Date Day Temp. ${ }^{\circ} \mathrm{F}$.
1912 a.m.

7. $x .1101 \cdot 2$. 10 infected $R$. appendiculatus nymphs were placed on the calf

$101 \cdot 6$

$2 \quad 101 \cdot 8$

$3101 \cdot 6101 \cdot 2$

$101 \cdot 8$

$\begin{array}{lll}4 & 102 & 101 \cdot 6\end{array}$

$\begin{array}{lll}5 & 101 \cdot 4 & 101 \cdot 8\end{array}$

$\begin{array}{lll}6 & 101 \cdot 6\end{array}$

$\begin{array}{lll}7 & 102.8\end{array}$

$8 \quad 102 \quad 102 \cdot 2$

$9100 \cdot 8^{101 \cdot 4}$

$10 \quad 100 \cdot 4^{100 \cdot 8}$

$11 \quad 101.2$

$12 \quad 101.8{ }^{103.8}$

$13101 \cdot 6102 \cdot 6$

$101 \cdot 6$

$\begin{array}{lll}14 & 101.8 & 103.2\end{array}$

$15 \quad 104 \cdot 4{ }_{105 \cdot 4}^{103 \cdot 2}$ Fever begins

$16 \quad 105 \cdot 4$

$17 \quad 106$

$106 \cdot 2$

$\begin{array}{lll}18 & 106.8\end{array}$

$\begin{array}{llll}19 & 107 & 106 \cdot 6 & \text { Very few parasites found }\end{array}$

$20 \quad 105.8$ 106.8 " "

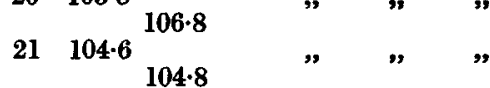

$\begin{array}{lll}22 & 104 & 3.6 \%\end{array} \%$ of r.b.c. infected

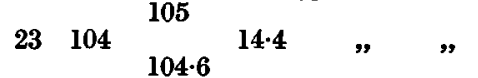

$24 \quad 104.8{ }_{106.2}^{104 \cdot 6} 20 \cdot 4 \quad " \quad$ "

$25105 \begin{array}{lllll}106 \cdot 2 & & & & \\ & & 105 \cdot 2 & & \end{array}$

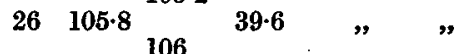

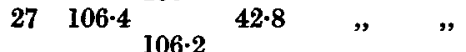

$28 \quad 106 \cdot 406 \cdot 2$

43.6 " ,

4. $x 1.29-100.2$ Calf died at 5.30 a.m.

\begin{tabular}{cc}
\multicolumn{2}{c}{ Treatment } \\
Eosin + sod. \\
bicarb. \\
solution. \\
Doos \\
$\begin{array}{c}\text { Doamin. } \\
\text { Dose }\end{array}$ & $\begin{array}{c}\text { c.c. } \\
3 \text { c.c. }\end{array}$ \\
3 c.c. & 2 c.c. \\
10 c.c. & 2 c.c. \\
13 c.c. & 3 c.c. \\
10 c.c. & 3 c.c. \\
8 c.c. & 4 c.c.
\end{tabular}


68 Drug Treatment of East Coast Fever of Cattle

\section{Experiment VIII (Cow 4).}

Treatment: "606," 2 grammes given intravenously in one dose. Weight of cow 1008 lbs.

Date Day Temp. ${ }^{\circ} \mathbf{F}$
1911

18. $\Pi$. 1 - 12 infected $R$. appendiculatus $(6 \AA, 6$ o $)$ were placed upon the $2^{100 \cdot 8^{100 \cdot 6}}$

$3 \quad 100 \cdot 6^{100 \cdot 6}$

$100 \cdot 8$

$101 \cdot 2$

$\begin{array}{lll}5 & 100 \cdot 8 & 101\end{array}$

$6 \quad 101 \cdot 2$

$7^{101 \cdot 4^{100 \cdot 6}}$

$8 \quad 100 \cdot 8^{101}$

y $100 \cdot 6100 \cdot 4$

$10101 \quad 101$

$101 \cdot 2$

$11 \quad 101 \cdot 8$

$12 \quad 101 \cdot 4^{101 \cdot 6}$

$13 \quad 104 \quad 101.6 \quad$ Fever begins

14102

$\begin{array}{lll}15 & 104 & 103\end{array}$

$16 \quad 101 \cdot 6$

$101 \cdot 8$

$17 \quad 103-4$

$18 \quad 103 \cdot 4$

$19 \quad 105 \cdot 2104 \cdot 6$

$20105.4{ }^{105}$ M. $0.4 \%$ of r.b.c. contain parasites

$21 \quad 104.4 \begin{array}{lllll}105 \cdot 2 & \text { E. } 0 \cdot 8 & 0.8 & & \end{array}$

$21 \quad 104 \cdot 4 \quad 104 \cdot 2$ E. $4 \cdot 0 \quad$ " " " "

22103.6 at 10 a.m. $6.4 \%$ of r.b.c. contain parasites 3 p.m. 8.4 , " , " at time when treated with " 606 " intra. venously $5, \quad 8.4 \quad$, $\quad$, $\quad, \quad, \quad 2$ hrs later $\begin{array}{cccc}104 \cdot 4 & 7 & 7 & 8 \cdot 4 \\ & 9 & & 8 \cdot 8\end{array}$

$23 \quad 101 \cdot 4 \quad 11$ a.m. $21 \cdot 0$

",

$23 \quad 101 \cdot 498.2 \quad$ a.m. $21 \cdot 0 \quad "$,

13. III. 24 - Cow found dead at 6 a.m. 
Experiment IX (Calf 15).

Treatment: Emetine hydrochloride, $1 \%$ in $\mathrm{NaCl}$ solution given intravenously in doses ranging from 1 to 10 c.c. on 9 successive days.

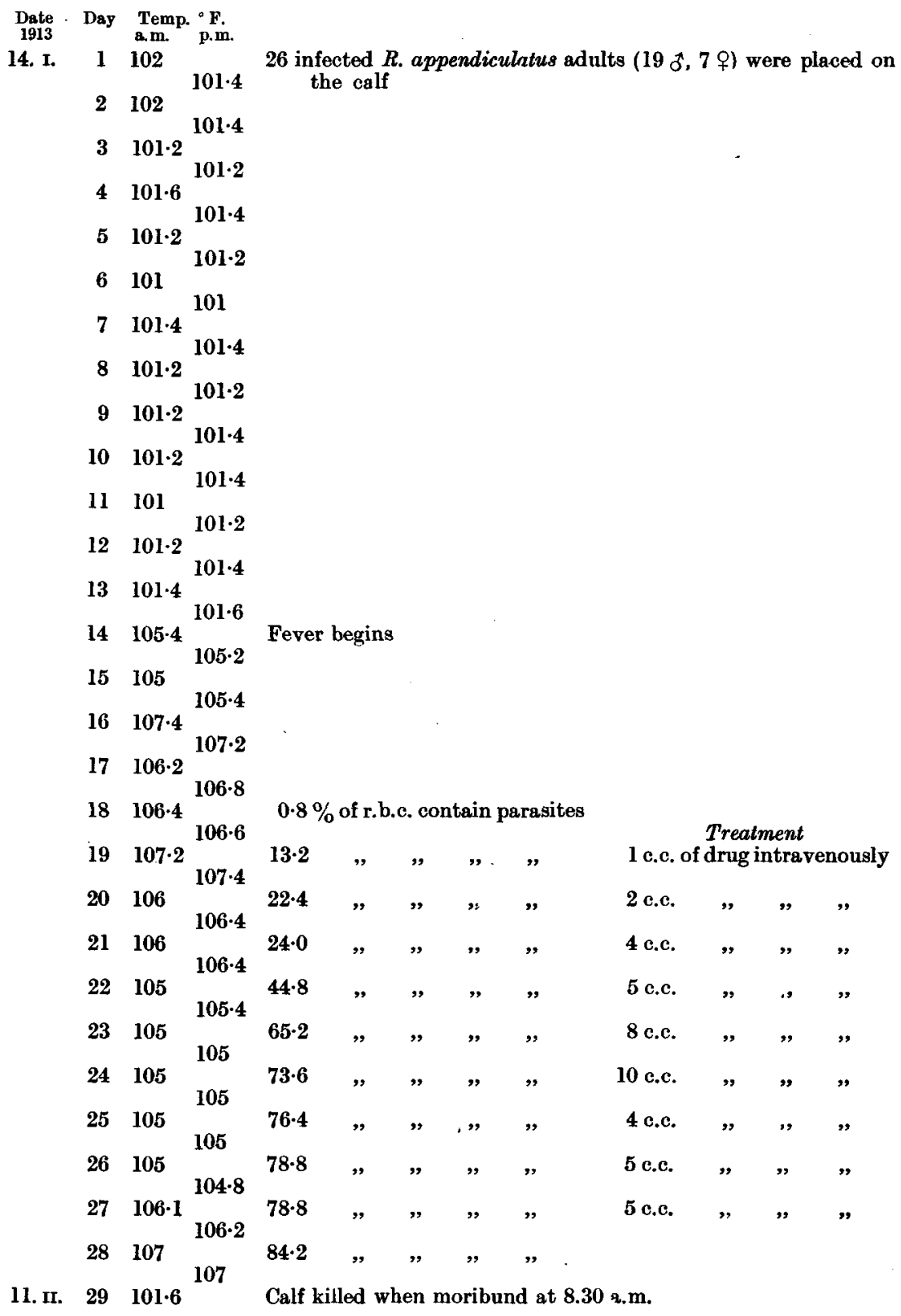


70 Drug Treatment of East Coast Fever of Cattle

\section{EXPeriment X (Calf 9).}

Treatment: Mercury salicylate, $1 \%$ in $0.8 \% \mathrm{NaCl}$ solution given intravenously on 9 days in doses ranging from 4 to 20 c.c.

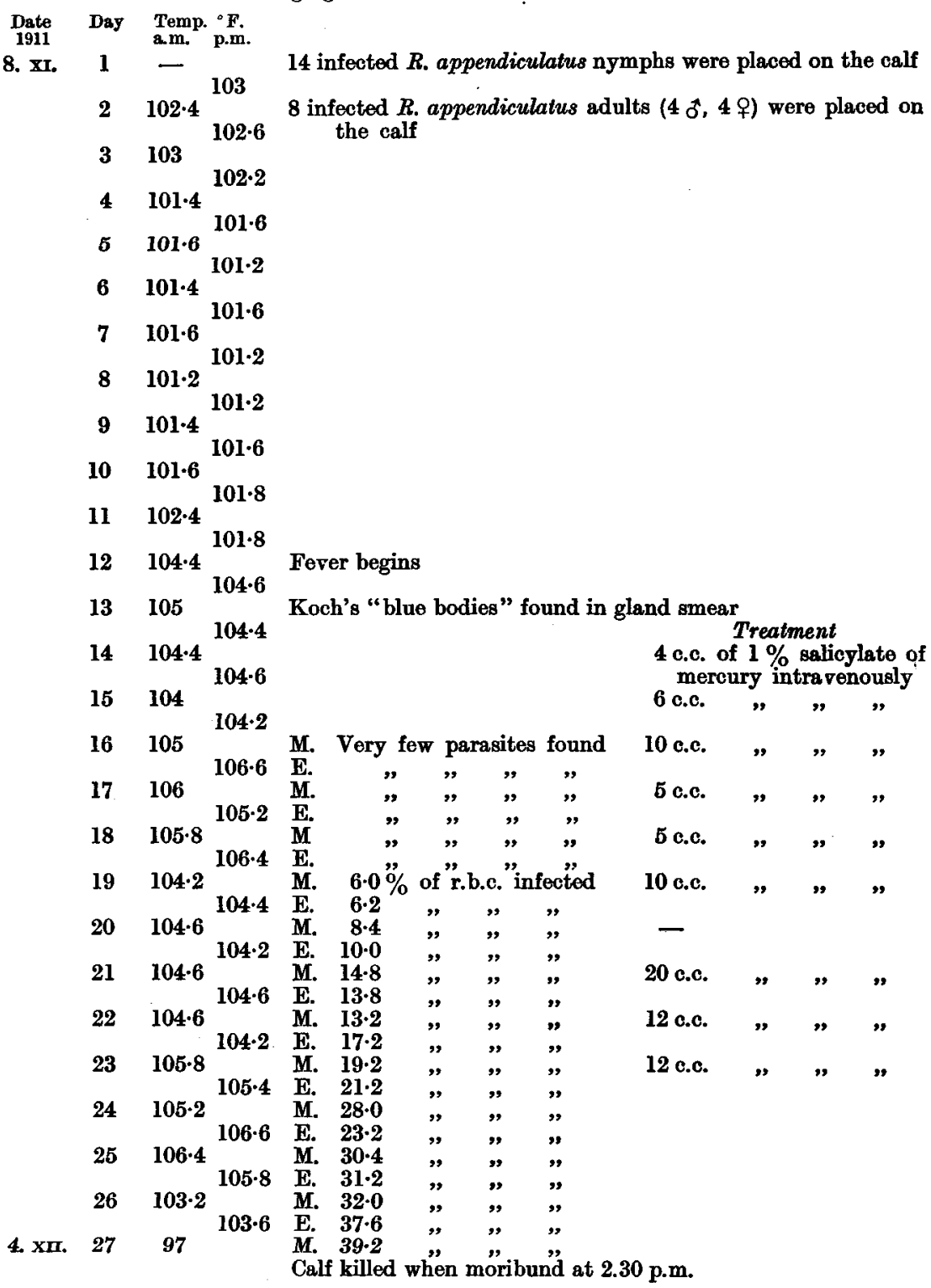




\section{EXPERIMENT XI (Calf 10).}

Treatment: Mercury succinimide in $3 \%$ solution given intravenously, 2,4 and 3 c.c. being injected on the 15th, 16th and 19th days respectively.

Date Day Temp. ${ }^{\circ} \mathrm{F}$.
1912

30. I. $1-12$ infected $R$. appendiculatus nymphs were placed on the calf

$2 \quad 101 \cdot 4$

$101 \cdot 4$

$3101 \cdot 6$

$4101 \cdot 2$

$5101^{101 \cdot 4}$

$6101 \cdot 4^{101 \cdot 2}$

101

$\begin{array}{llll} & 101 \cdot 2 & & \\ & & 102\end{array}$

$8 \quad 101 \cdot 6$

$9 \quad 101.8$

$10101 \cdot 4^{101 \cdot 2}$

$101 \cdot 8$

$11 \quad 103 \quad 103.2$ Fever begins

$12 \quad 103 \cdot 2$

$13 \quad 104 \cdot 6^{103 \cdot 8}$

$14 \quad 104 \cdot 8$ 104.6

$15 \quad 104 \cdot 8{ }_{105}^{104 \cdot 4}$ Very few parasites found 2 c.c. of drug given intravenously

$16 \quad 105.2$

$17^{104 \cdot 4^{105 \cdot 4}} \quad 0.4 \%$ of r.b.c. infected

$18 \quad 102 \cdot 6$

$19 \quad 102 \cdot 6$

$20100 \cdot 6$

$21 \quad 100 \cdot 6$

$100 \cdot 6$

$100 \cdot 2$

$\begin{array}{llllll}23 & 99 \cdot 6 & & 20 \cdot 8 \quad, \quad \text {, }\end{array}$

$2499.4^{99.8} 15.6 \quad$ "

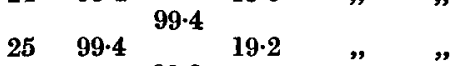

$26 \quad 99.4 \begin{array}{lllll}99 \cdot 2 & & & & \\ & & 24.0 & \end{array}$

$\begin{array}{lllll}27 & 98.6 & 99 \cdot 2 & 23.4 & \end{array}$

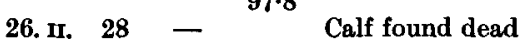




\section{Drug Treatment of East Coast Fever of Cattle}

\section{EXPERIMENT XII (Calf 5).}

Treatment: Quinine bihydrochloride, 55-60 grains dissolved in 25 c.c. of water, injected subcutaneously at 11 a.m. on 14 successive days. (Prepared as 5 grain tabloids.)

Date Day Temp. ${ }^{\circ} \mathbf{F}$.

25. Iv. $1 \quad 101 \cdot 212$ infected $R$. appendiculatus adults $(6,6$, $\$$ ) were placed on the calf 2 101.4 Ncte: it is not clear which lot of ticks produced infection,

3101 possibly it may have been one of those following

4 101.5 16 infected $R$. appendiculatus nymphs were placed on the calf

$5 \quad 101 \cdot 2$

$6 \quad 101 \cdot 8$

$7 \quad 102 \cdot 3$

$8 \quad 101 \cdot 8$

$9 \quad 101 \cdot 2$

$10 \quad 101 \cdot 3$

$11 \quad 100 \cdot 6$

12101

$13 \quad 101 \cdot 5$

$14101 \cdot 6$

15101

$\begin{array}{ll}16 & 102\end{array}$

$\begin{array}{lll}17 & 102 \cdot 1\end{array}$

18102

$19 \quad 101 \cdot 5$

$20 \quad 102 \cdot 1$

21101

$22 \quad 100 \cdot 6$

$23 \quad 101.5$

$24 \quad 102-1$

$25 \quad 101.3$

$26 \quad 101 \cdot 2$

27 101.4

some

some

some

(1)

Temp. $\circ$ F

a.m. p.m.

$28 \quad 103 \quad 104 \cdot 4$

$29 \quad 104 \cdot 6$

101.8

$\begin{array}{lll}30 & 101 \cdot 4 & 102 \cdot 2\end{array}$

$31 \quad 100 \cdot 2$

$32 \quad 100 \cdot 6$

$33 \quad 100 \cdot 6$

$101 \cdot 4$

$34 \quad 101.8$

$35 \quad 100 \cdot 8$

$101 \cdot 4$

$36 \quad 100 \cdot 1$

$37 \quad 101$

$\begin{array}{ll}38 & 101\end{array}$

$39101 \cdot 6$ 


\begin{tabular}{|c|c|c|c|c|c|c|c|c|c|c|}
\hline \multirow{3}{*}{$\begin{array}{l}\text { Date } \\
\text { 1911 } \\
\text { 3. VI. }\end{array}$} & \multicolumn{10}{|c|}{ EXPERIMENT XII (cont). } \\
\hline & Day & $\begin{array}{l}\text { Temp } \\
\text { a.m. }\end{array}$ & $\stackrel{\circ}{p} \mathbf{F}$ & & & & & & & \\
\hline & 40 & 104 & & \multicolumn{7}{|c|}{ Fever begins } \\
\hline & & & $105 \cdot 2$ & \multirow{2}{*}{\multicolumn{7}{|c|}{ Very fow parasites found }} \\
\hline & 41 & $105 \cdot 8$ & & & & & & & & \\
\hline & 42 & $104 \cdot 8$ & 1002 & & & , & \multirow{3}{*}{\multicolumn{4}{|c|}{$\begin{array}{c}\text { Treatment } \\
60 \text { grains quinine bihydrochloride }\end{array}$}} \\
\hline & & & $106 \cdot 8$ & \multirow{2}{*}{\multicolumn{3}{|c|}{ M. $\quad 0.8 \%$ of r.b.c. infected }} & & & & \\
\hline & 43 & $105 \cdot 2$ & & & & & & & & \\
\hline & & & $105 \cdot 2$ & E. $\quad 0 \cdot 8$ & , , & , & & & & \\
\hline & 44 & $105 \cdot 6$ & & M. $\quad 0.8$ & ", & , & 60 & ", & ", & , \\
\hline & & & $105 \cdot 8$ & E. $\quad 1 \cdot 2$ & $"$, & , & & & & \\
\hline & 45 & $104 \cdot 8$ & & M. $\quad 0 \cdot 4$ & , & $"$ & 60 & " & , & "’ \\
\hline & & & $104 \cdot 8$ & E. $\quad 3 \cdot 2$ & $"$ & $"$ & & & & \\
\hline & 46 & $105 \cdot 6$ & & $\begin{array}{ll}\text { M. } & 3 \cdot 2 \\
\mathbf{E} & \mathbf{3 \cdot 2}\end{array}$ & $"$ & $"$ & 60 & " & $"$ & $"$ \\
\hline & 47 & $104 \cdot 8$ & 100 & M. $4 \cdot 4$ & $"$ & $"$, & 60 & " & " & , \\
\hline & & & $103 \cdot 2$ & E. $\quad 6 \cdot 0$ & , & , & & 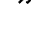 & & \\
\hline & 48 & $104 \cdot 2$ & & M. $\quad 8 \cdot 8$ & $"$ & ", & 60 & ", & $"$ & $"$ \\
\hline & 49 & & $103 \cdot 4$ & $\begin{array}{ll}\mathbf{E} . & \mathbf{4} \cdot \mathbf{8} \\
\mathbf{M} & \mathbf{3 \cdot 2}\end{array}$ & " & " & & & & \\
\hline & $\mathbf{4 9}$ & 102 & $101 \cdot 8$ & $\begin{array}{ll}\text { E. } & \mathbf{3} \cdot \mathbf{2} \\
\text { E. }\end{array}$ & ", & 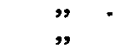 & 60 & $"$ & "' & $"$ \\
\hline & 50 & 100 & & M. $13 \cdot 2$ & " & $"$ & 55 & $"$ & "' & $"$ \\
\hline & 51 & $102 \cdot 6$ & 102 & $\begin{array}{l}\text { E. } 11 \cdot 6 \\
\text { M. } 15 \cdot 6\end{array}$ & $"$ & $"$ & 60 & & & \\
\hline & & & $102 \cdot 6$ & E. $18 \cdot 0$ & $\ddot{~}$ & ", & ov & $"$ & : & ", \\
\hline & 52 & 101 & & M. $16 \cdot 0$ & " & ", & 60 & $"$ & "' & $"$ \\
\hline & 53 & 102 & 1042 & M. $24 \cdot 0$ & " & "' & 60 & " & ", & " \\
\hline & & & $103 \cdot 8$ & E. $25 \cdot 2$ & $"$ & " & & & & \\
\hline & 54 & 103 & & M. $24 \cdot 0$ & " & " & 60 & ", & $"$ & $\because$ \\
\hline & 55 & $103 \cdot 6$ & 100 & $\begin{array}{l}\text { E. } 21 \cdot 6 \\
\text { M. } 29 \cdot 6\end{array}$ & "” & $"$ & 60 & $\because$ & ". & " \\
\hline & & & $105 \cdot 2$ & E. $33 \cdot 2$ & ", & $"$ & & 3 & 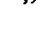 & $" 7$ \\
\hline 19. VI. & 56 & $\cdot 102 \cdot 6$ & & M. 31.2 & " & ", & 60 & $"$ & ", & $"$ \\
\hline & & & $101 \cdot 8$ & Calf kille & Th & moribund & at 6.1 & m. & & \\
\hline
\end{tabular}




\section{Drug Treatment of East Coast Fever of Cattle}

\section{Experiment XIII (Calf 4).}

Treatment: Quinine hydrochloride, $30-45$ grains in 20 c.c. of water injected intramuscularly at 10.45 a.m. on 5 days ( 5 grain tabloids) and Quinine bihydrochloride, ditto on 7 days.

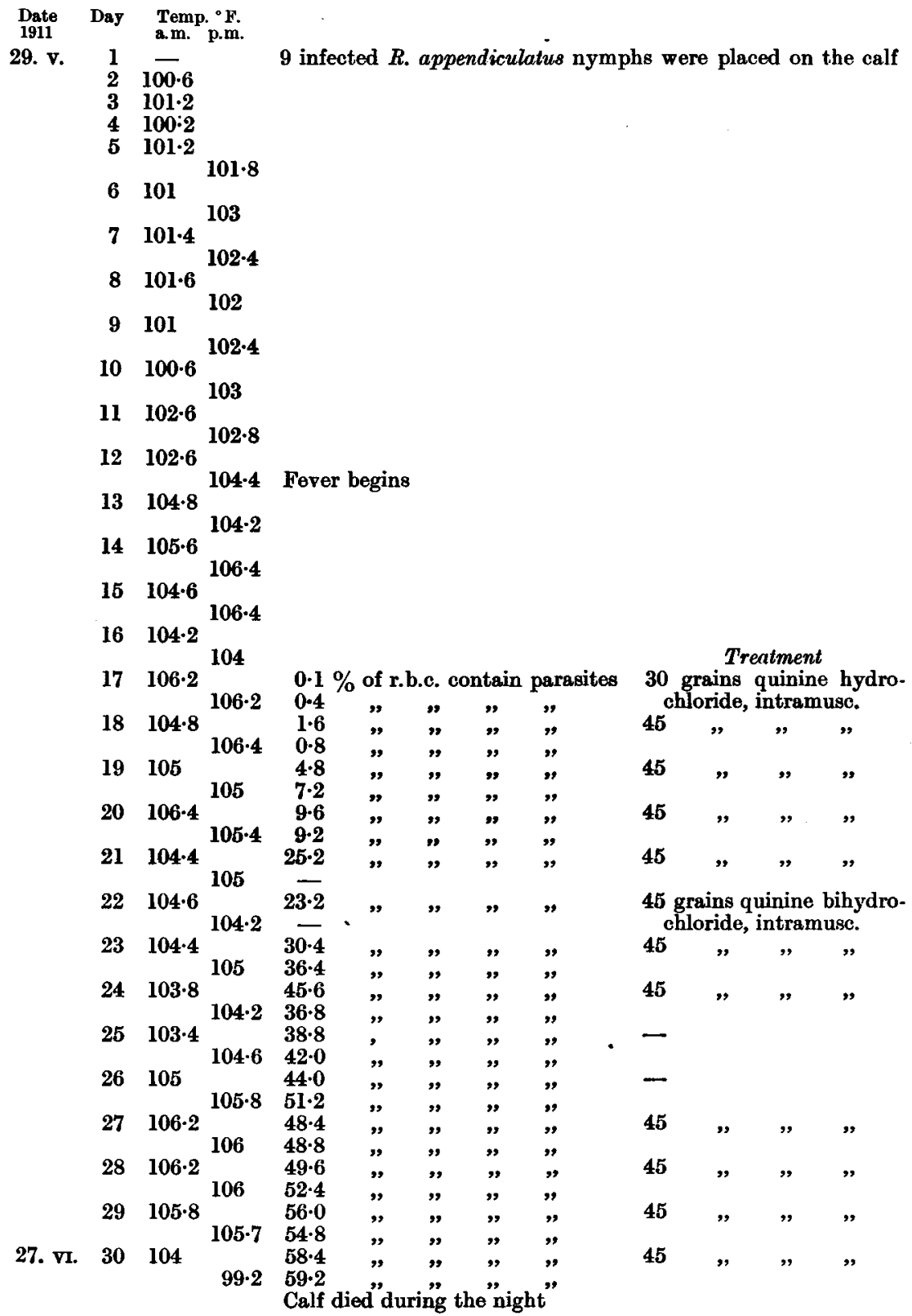




\section{EXPERIMENT XIV (Calf 22).}

Treatment: Ethylhydrocupreine $\mathrm{HCl}$, subcutaneous injection of 24 c.c. of an $8 \%$ solution on each of five successive days, beginning on the second day after the appearance of parasites in the calf's blood.

Date Day Temp. ${ }^{\circ} \mathrm{F}$.

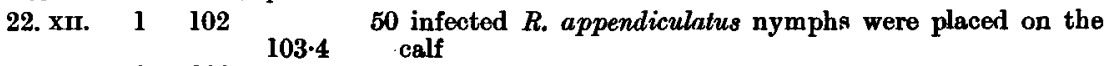

$\begin{array}{lll}2 & 101 & 103.4 \\ & & 102.8\end{array}$

$3 \quad 101 \cdot 4 \underset{102 \cdot 8}{102 \cdot 8}$

$4 \quad 101 \cdot 6 \quad 102 \cdot 6$

$5 \quad 102 \cdot 4$

6 $101 \cdot 6^{102}$

$7 \quad 101 \cdot 102$

$8 \quad 101 \cdot 102 \cdot 2$

$9 \quad 101.8103$

$102 \cdot 4$

$10 \quad 102 \cdot 6,102$

$11 \quad 101.8 \underbrace{102}_{103.6}$ Fever begins

$12 \quad 101 \cdot 6$

$13 \quad 103 \cdot 4^{103 \cdot 6}$

14104

$14 \quad 102 \cdot 8$

$15 \quad 105 \cdot 3$

$16 \quad 104 \cdot 8$

$17 \quad 105$

$17 \quad 104 \quad 105 \cdot 2$

$\begin{array}{lll}18 & 105 & 105 \cdot 4\end{array}$

$\begin{array}{llll}19 & 106 & 105 \cdot 4 & \text { Very few parasites found }\end{array}$

2010524 c.c. of drug subcutaneously

$21 \quad 104 \cdot 2^{10}$

$22 \quad 102 \cdot 2^{102}$

$23 \quad 101 \cdot 6$

24

$03 \cdot 2$

$\begin{array}{lll}24 & 102 \cdot 6 & \\ & & 103 \cdot 2\end{array}$

$25 \quad 103 \cdot 4$

16. I. $26 \quad 104.6$ $104 \cdot 6$ 1912

$36-0$
Calf killed" at 12.20 p.m.

24 c.c. , , ,

24 c.c.

24 e.c.

24 c.c. 


\section{EXPERIMENT XV (Calf 16).}

Treatment: Ammonium fluoride, $0.5 \%$ in $0.8 \% \mathrm{NaCl}$ solution injected in doses of 18-20 c.c. intravenously on 9 successive days.

$\begin{array}{lll}\text { Date Day } & \text { Temp. } \\ 1913 & & \\ \text { a.m. } & \text { p.m. }\end{array}$

4. III. $1-50$ infected $R$. appendiculatus nymphs were placed on the calf

$\begin{array}{llr}2 & 102 \cdot 2 & 102 \cdot 2 \\ 3 & 102 \cdot 2 & 102 \cdot 4\end{array}$

$102 \cdot 6$

$\begin{array}{lll} & 101 \cdot 8 & \\ & 104 \cdot 6\end{array}$

$5 \quad 102.2$

$6^{102 \cdot 4^{102 \cdot 4}}$

$7^{102 \cdot 4^{102 \cdot 6}}$

$102 \cdot 4$

$8 \quad 102.4$

$9 \quad 102 \cdot 6$

$10102 \cdot 102 \cdot 4$

$11 \quad 103 \quad 102 \cdot 4$

$\begin{array}{lll}11 & 103 & 102 \cdot 6\end{array}$

$12 \quad 103 \cdot 6{ }_{103 \cdot 4}$ Fever begins

$13 \quad 103 \cdot 4$

$14 \quad 105 \quad 103 \cdot 6$

$15106 \cdot 2105 \cdot 2$

$106 \cdot 6$

$\begin{array}{ll}16 & 105.8\end{array}$

$17 \quad 107 \cdot 4$

A few parasites found

$18 \quad 104 \cdot 4$

$19105 \quad 104 \cdot 2$

$20 \quad 105 \cdot 4 \begin{array}{ll}105 \cdot 2 \\ 105 \cdot 8\end{array}$

$21 \quad 104.8$

$104 \cdot 6$

$22 \quad 104 \cdot 8 \quad 104 \cdot 6$

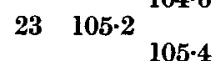

$24.106 \cdot 4 \quad \frac{105 \cdot 4}{106 \cdot 2}$

$\begin{array}{lll}25 & 107 & 107\end{array}$

$26 \quad 107 \cdot 4$

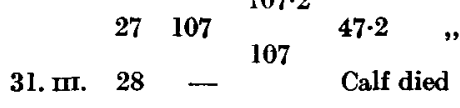

$107 \cdot 2$

$46.8 \%$ of r.b.c. infect. 20 c.c.

20 c.c. of ammonium fluoride sol. intrav.

20 c.c.

20 c.

20 c.c.

20 c.c

18 c.c.

20 c.c.

20 c.c.

43.6 ,, ,

, 


\section{Experiment XVI (Calf 20).}

Treatment: Potassium iodide in $25 \%$ solution, injected intravenously on 5 successive days, the dose rising from 20 to 40 c.c.

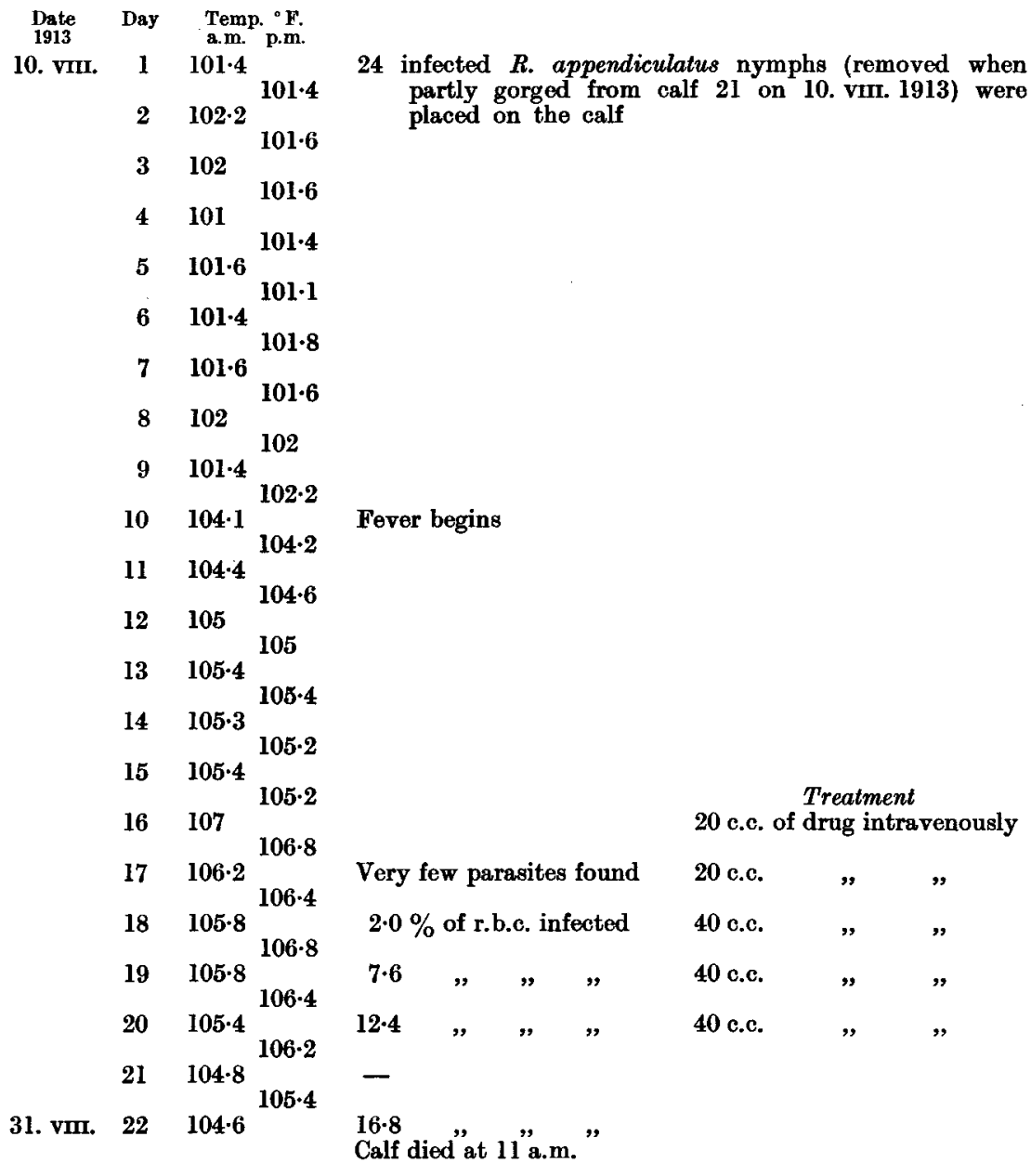




\section{Drug Treatment of East Coast Fever of Cattle}

\section{Experiment XVII (Calf 21).}

Treatment: Calcium lactate, in $10 \%$ solution, was injected intravenously on 7 successive days, the dose rising from 3 to 5 c.c.

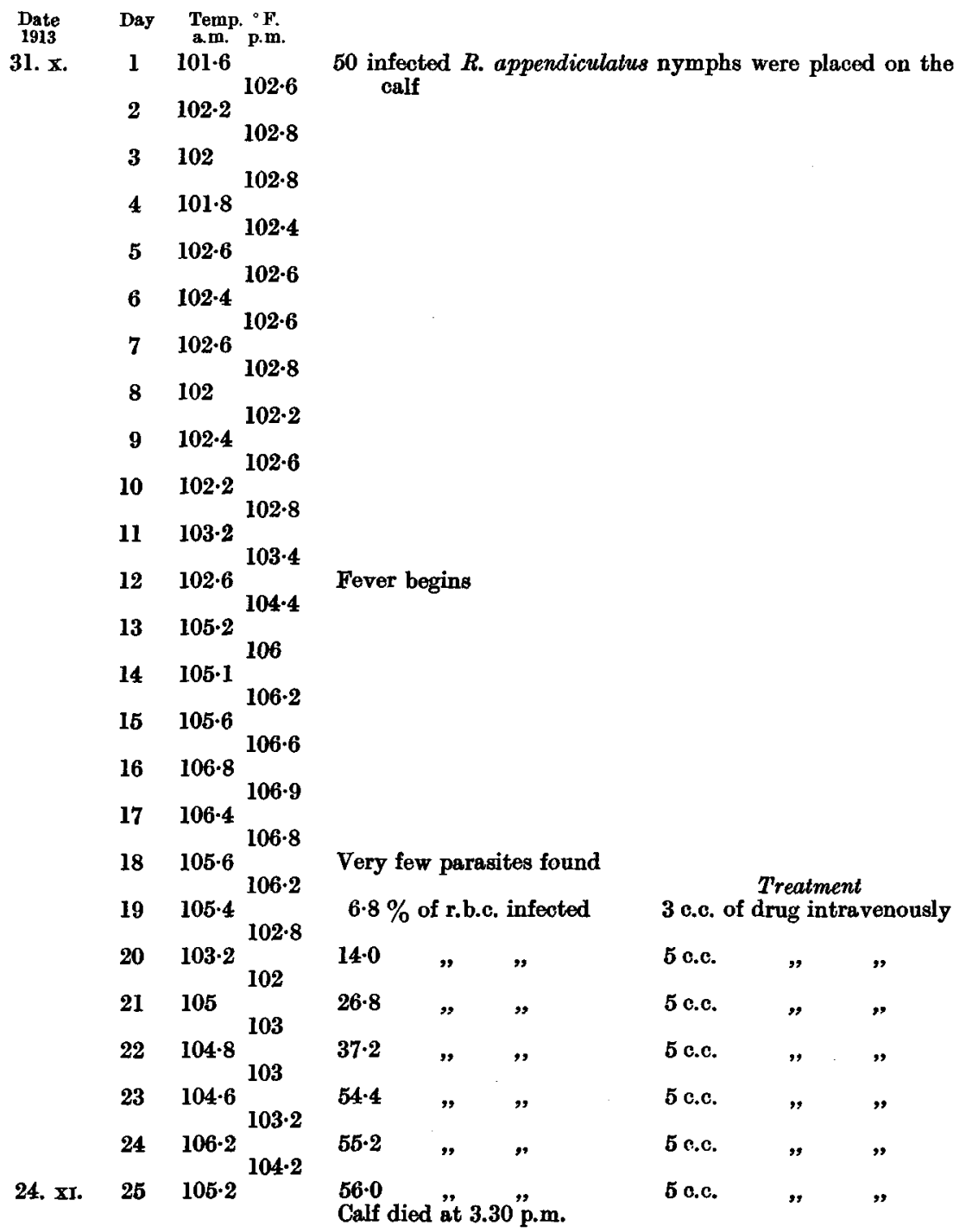




\section{EXPERIMENT XVIII (Calf 17).}

Treatment: Nuclein from thymus gland, 10 c.c. of a $5 \%$ solution given subcutaneously on 5 days.

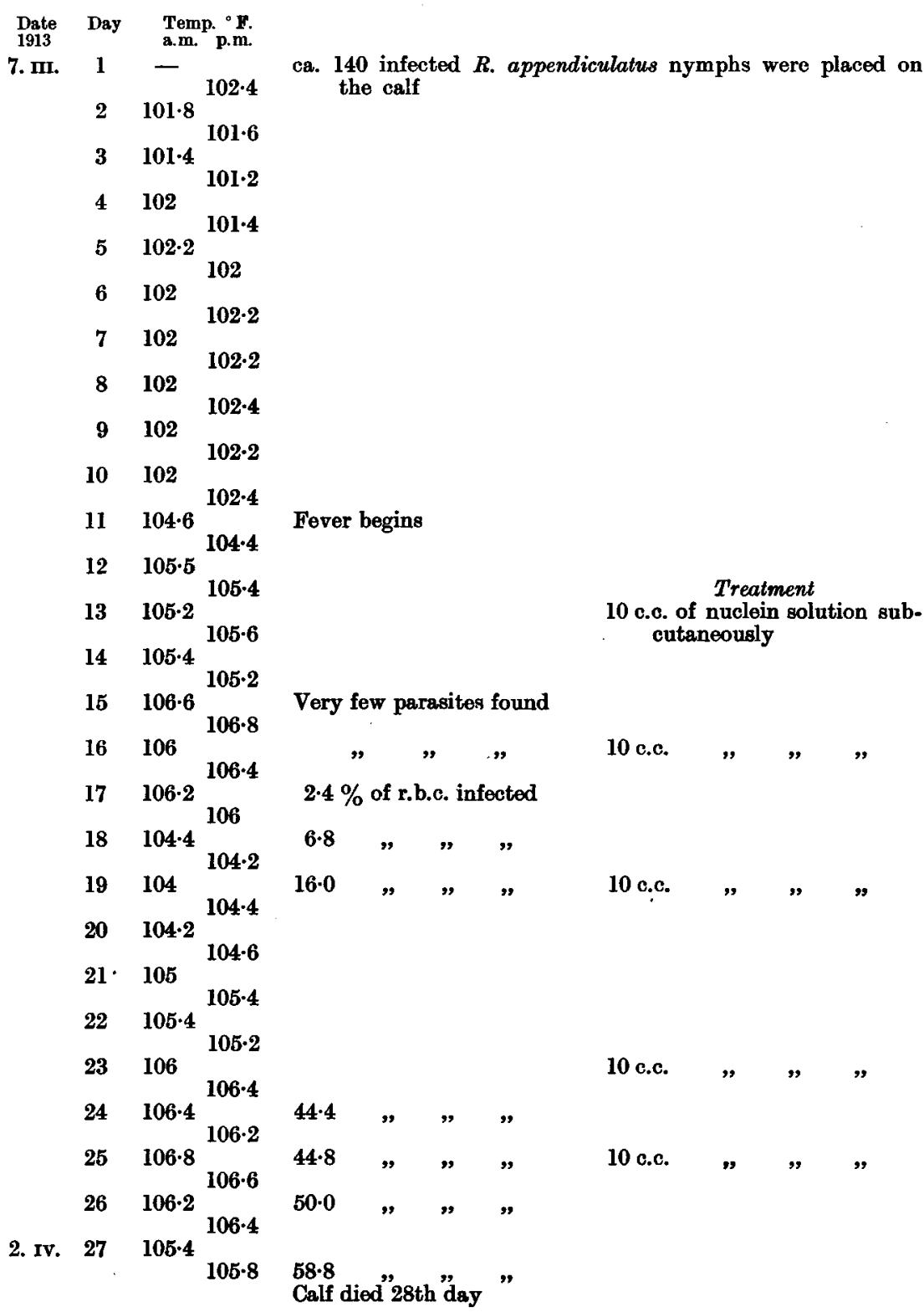


80 Drug Treatment of East Coast Fever of Cattle

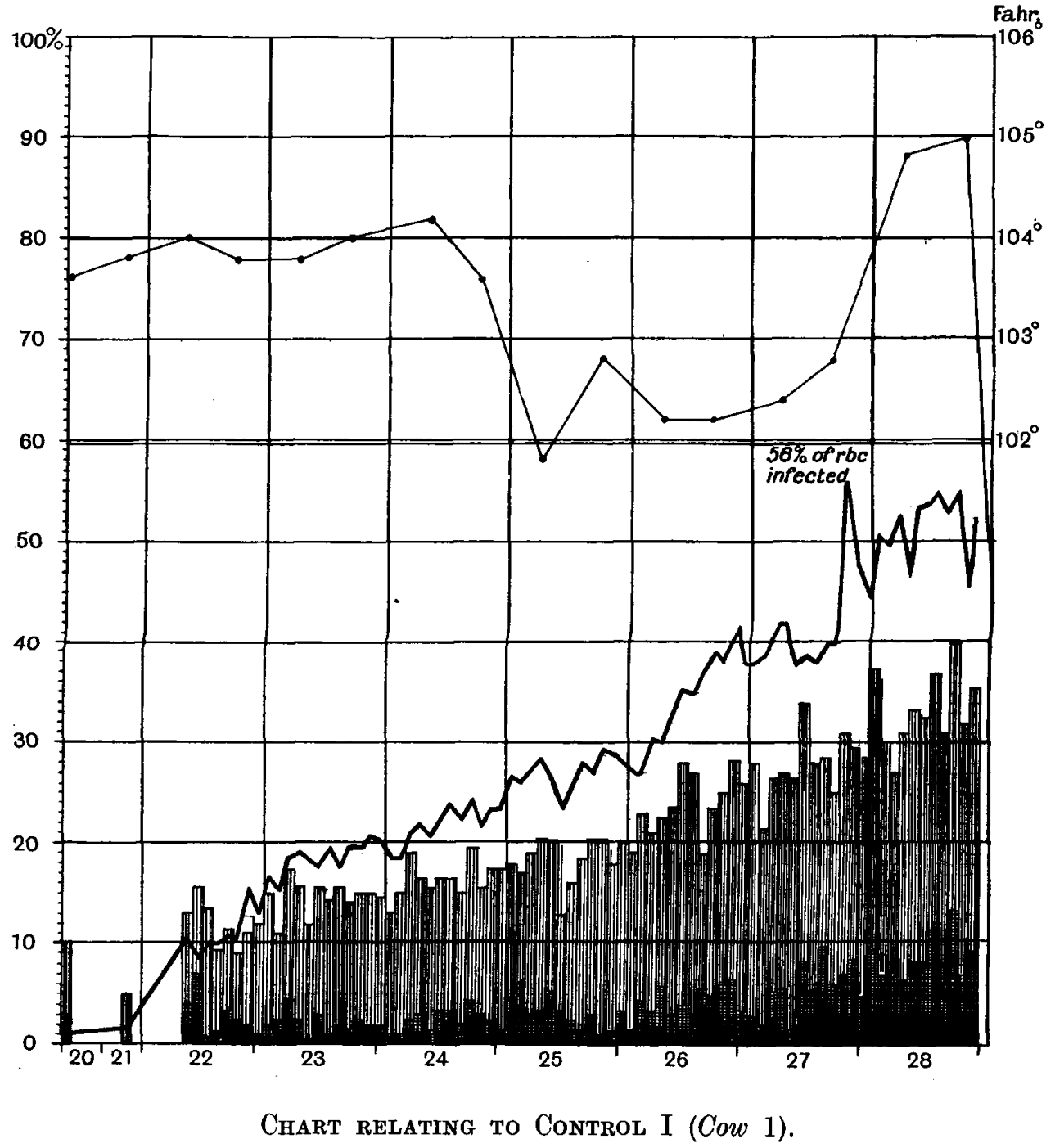

The body temperature is indicated by the thin line above. The increase in the percentage of infected red blood corpuseles is represented by the bold black line rising from $1.2 \%$ on the 20 th day after tick infestation to $56 \%$ on the 27 th day. The vertical columns represent the total number of infected corpuseles taken as 100 (corresponding to the percentage column on the left); the white part above each column represents the proportion of corpuscles containing a single parasite, the vertically, horizontally shaded, and black parts indicate respectively the proportion of corpuscles containing 2,3 and 4 or more parasites. The days of observation are recorded below. The blood films were prepared every two hours, day and night, starting on day 22. 
Records of Cattle which were not treated.

For the sake of comparison I append the protocols relating to three animals which were not subjected to treatment and which may be regarded as controls. In the case of Control I a very careful examination of the blood was made in respect to parasites, the results being given graphically in the accompanying chart.

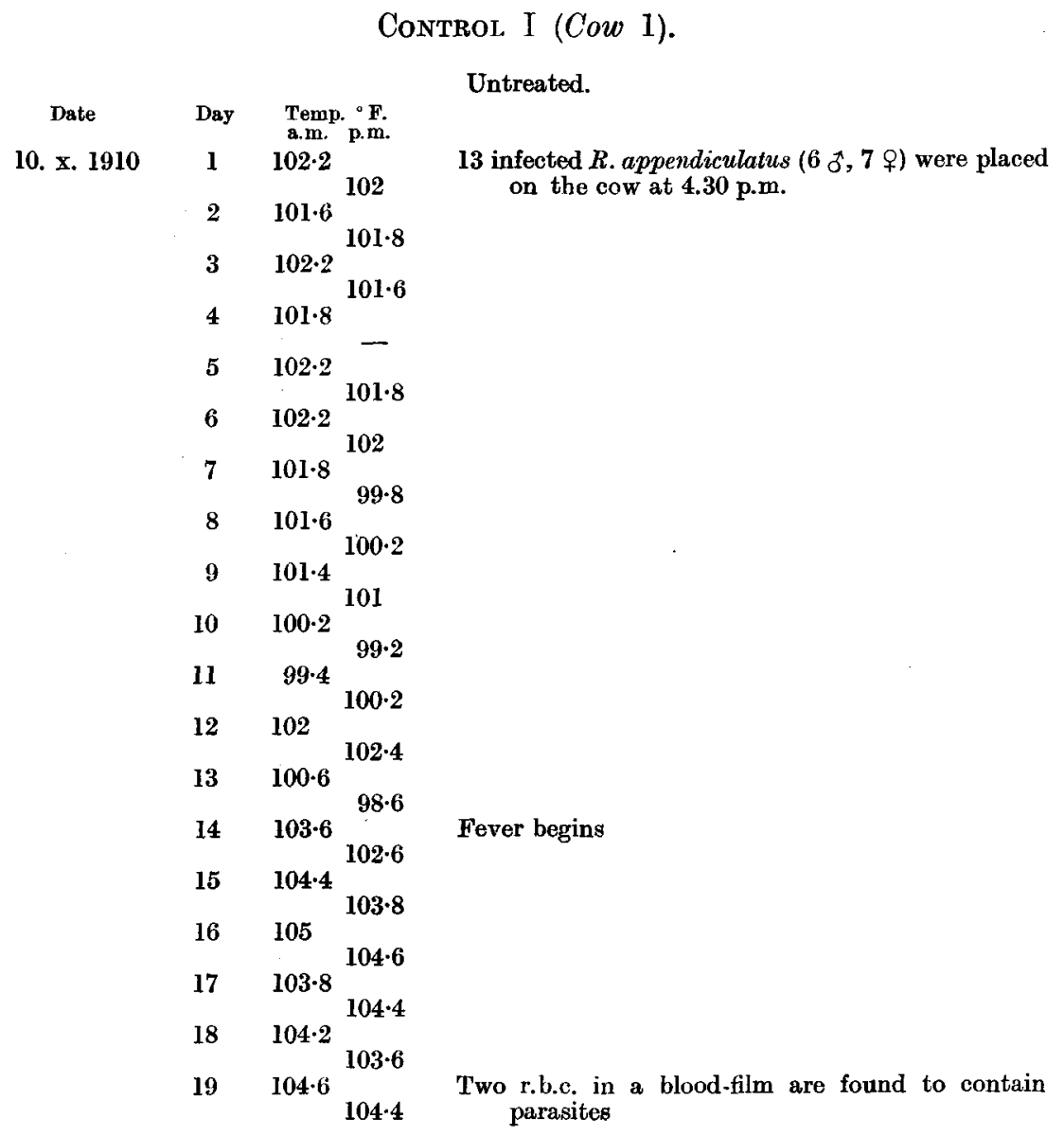


CONTROL I (cont.).

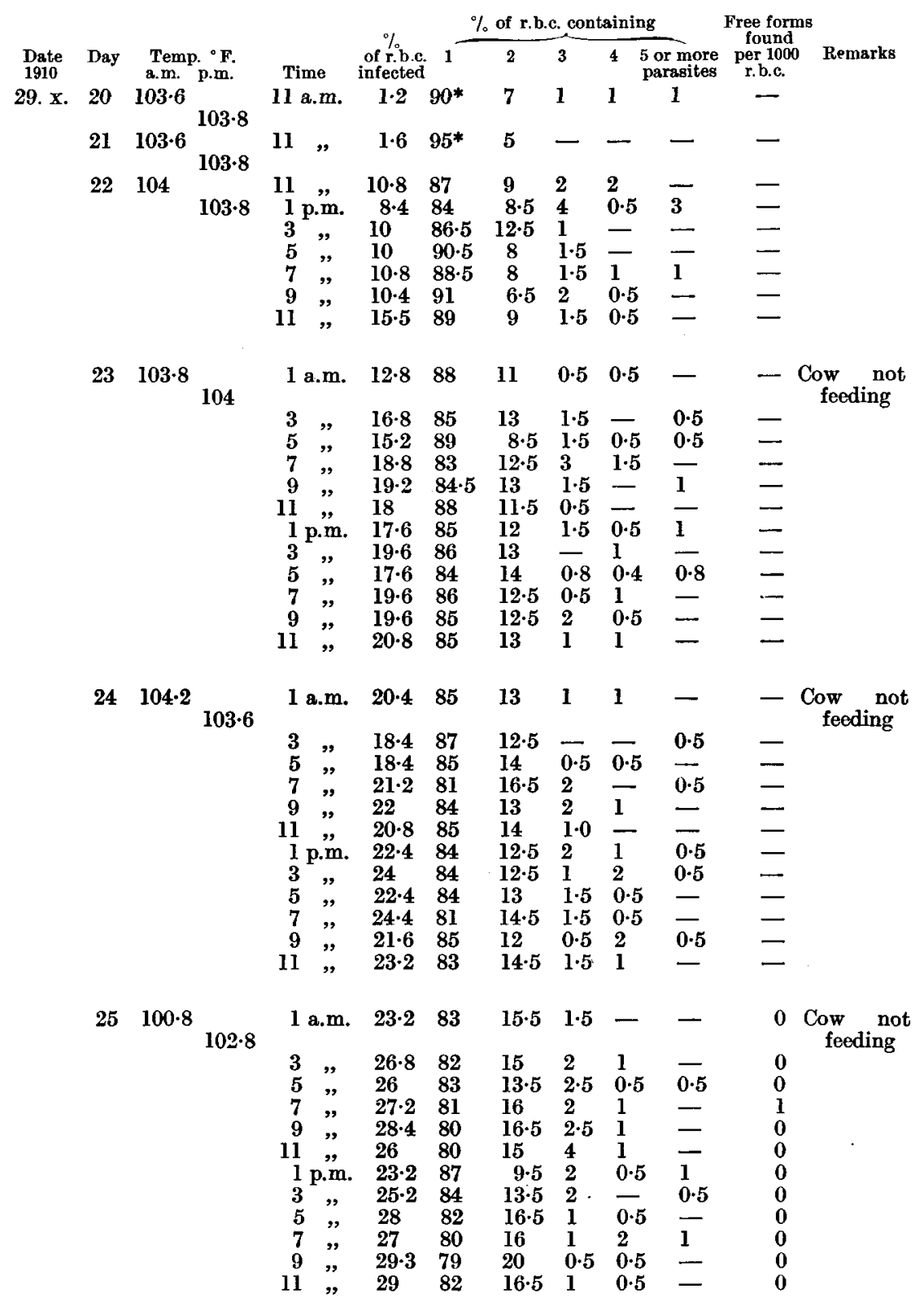

* 100 infected r.b.c. counted for types, 200 in the rest. 


\section{Control I (cont.).}

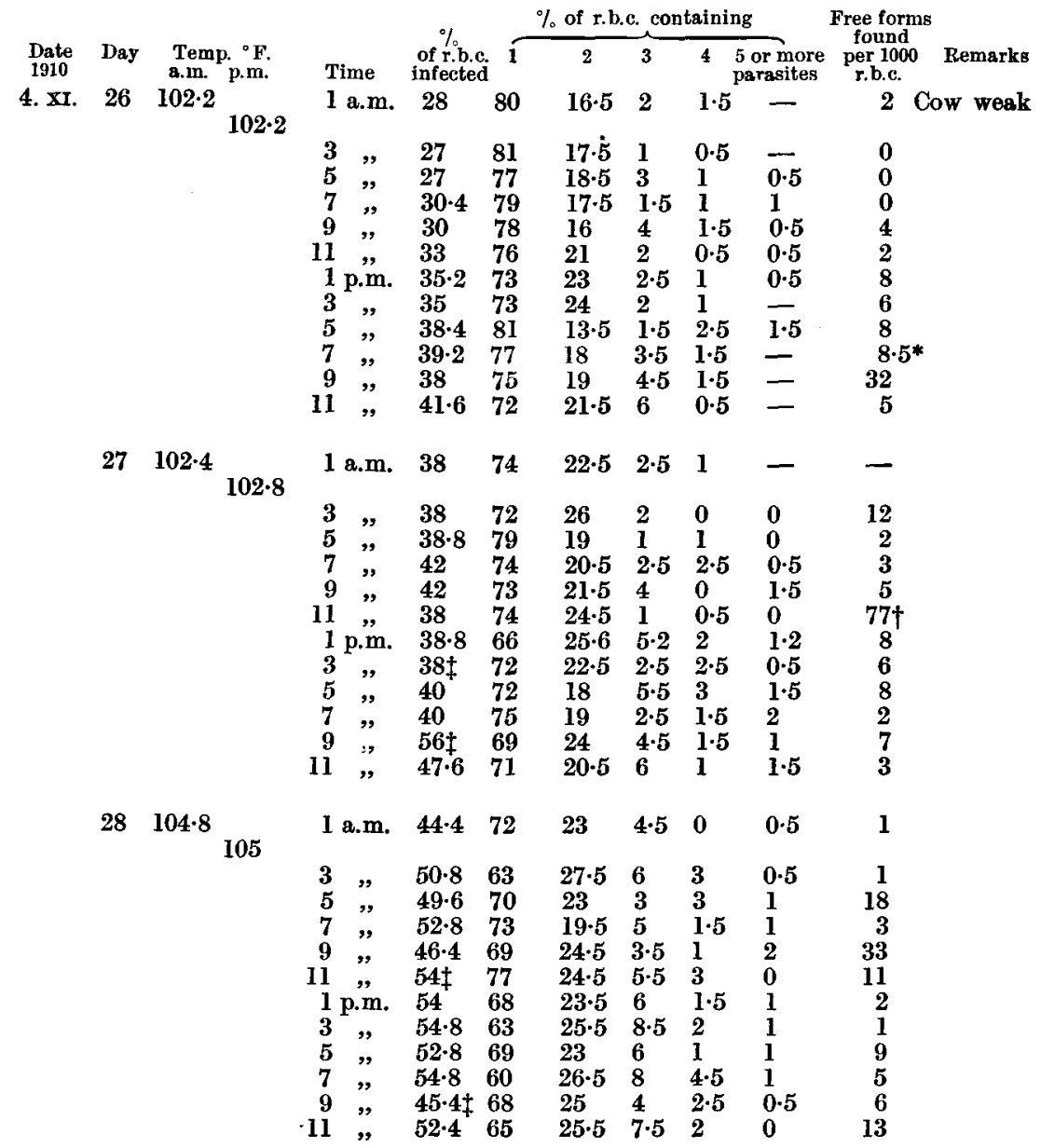

7. xI. $29 \longrightarrow$ l a.m. Cow found dead

* 2000 r.b.c. counted.

$\dagger$ Film very unequal, 2000 r.b.c. counted.

$\ddagger 500$ r.b.c. counted for $\%$ of infection, 250 counted otherwise. 
84 Drug Treatment of East Coast Fever of Cattle

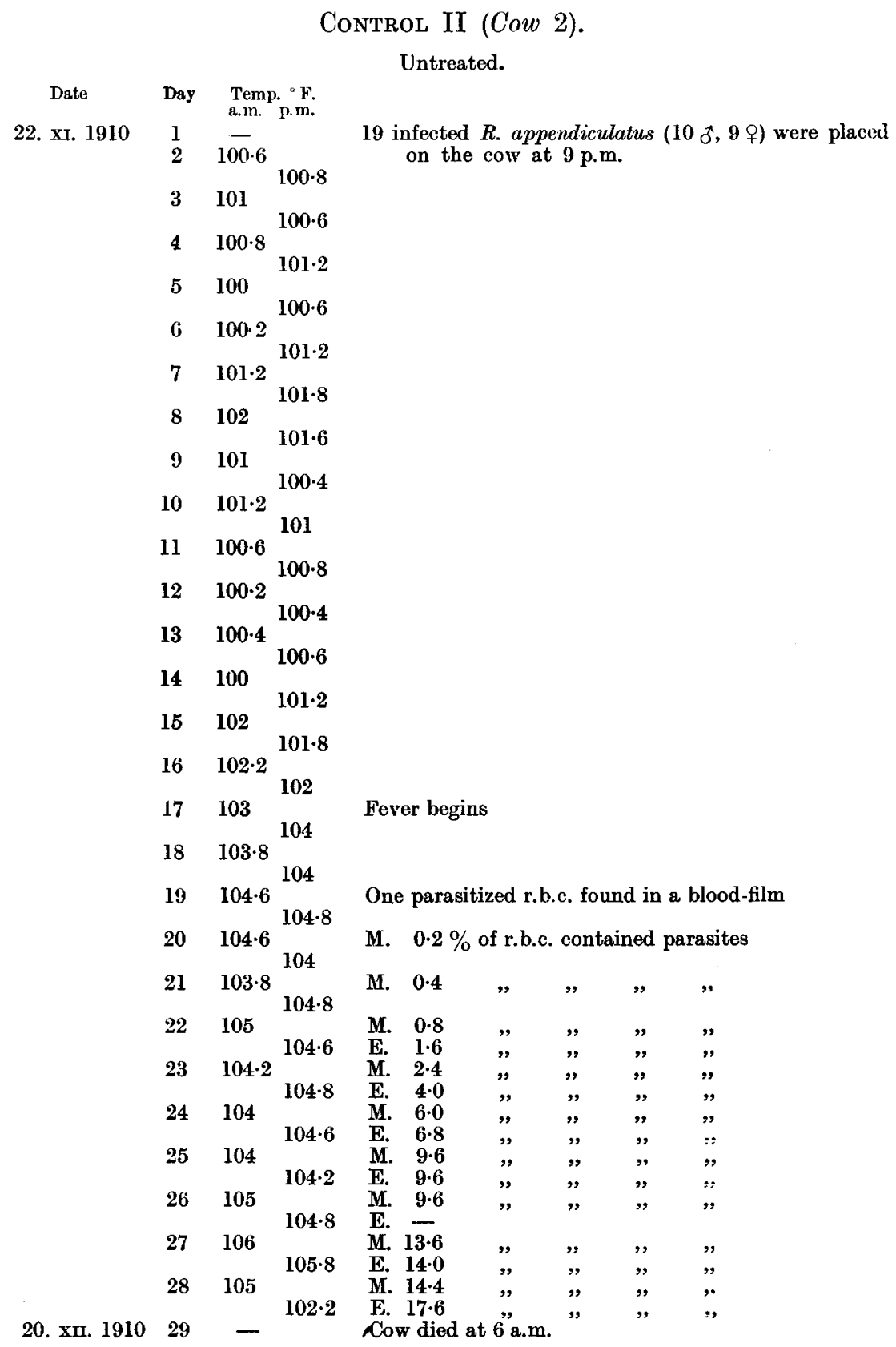


Control III (Calf 23).

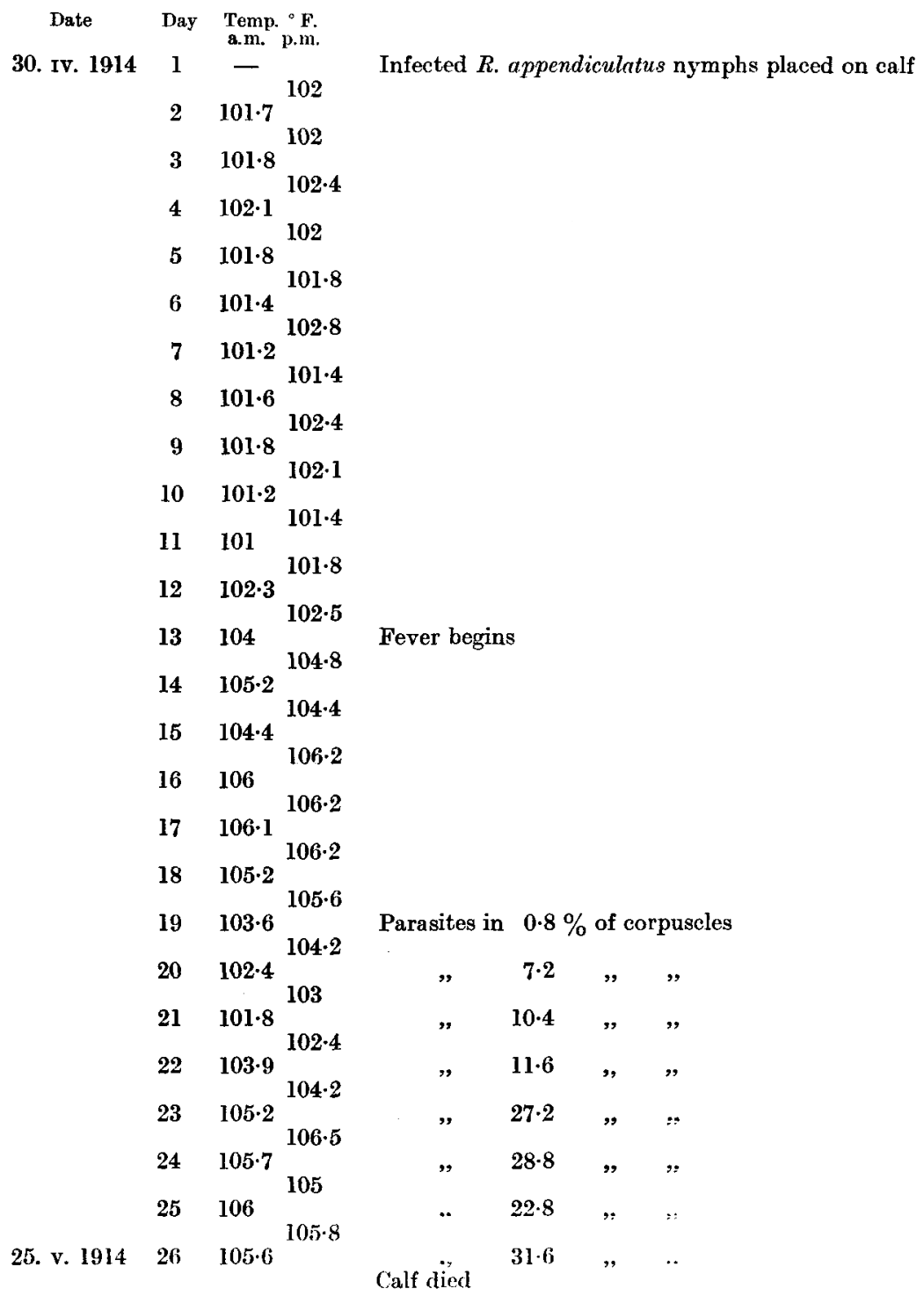




\section{Drug Treatment of East Coast Fever of Cattle}

\section{Summary aNd Conclusions.}

No drug has been found which will influence the fatal course of East Coast Fever or retard the multiplication of Theileria parva in the blood of the affected cattle.

The drugs which were tried with negative results were Trypanblue, Congo red, Tryposafrol, Creosote and Oleum copaivae, Arsacetin, Soamin, "606," Emetine hydrochloride, Mercury salicylate, Mercury succinimide, Quinine bihydrochloride and hydrochloride, Ethylhydrocupreine, Ammonium fluoride, Potassium iodide, Sodium salicylate, Calcium lactate, and Nuclein.

All of our animals died-18 treated and 3 untreated-and showed typical lesions at autopsy. They were all infected by means of ticks (Rhipicephalus appendiculatus) which had fed on infected cattle as larvae and nymphs and been placed on the experimental animals as nymphs and adults respectively.

The increase in the number of parasitized red blood corpuscles, but for slight irregularities, proceeds continuously night and day until the animal dies. We have not as yet observed a case ending in recovery.

The accompanying table gives a summary relating to each experimental animal (except XII): the number and kind of ticks which produced infection, the incubation period, the time when the parasites appeared in the peripheral blood, the time when the animals died, and the maximum percentage of parasitized blood corpuscles observed during the course of the disease. The days are all reckoned from Day 1 when the infective ticks were placed upon the cattle.

The expenses of this investigation were mainly defrayed by means of a grant made by the Parliament of the Union of South Africa.

My thanks are due to Dr W. E. Dixon, F.R.S., University Lecturer in Pharmacology, Cambridge, for kindly suggesting the trial of several of the drugs which were used. I am also indebted to my Laboratory Assistant, Mr B. G. Clarke, for much valuable help, especially in the making of the blood counts. 
G. H. F. Nuttall

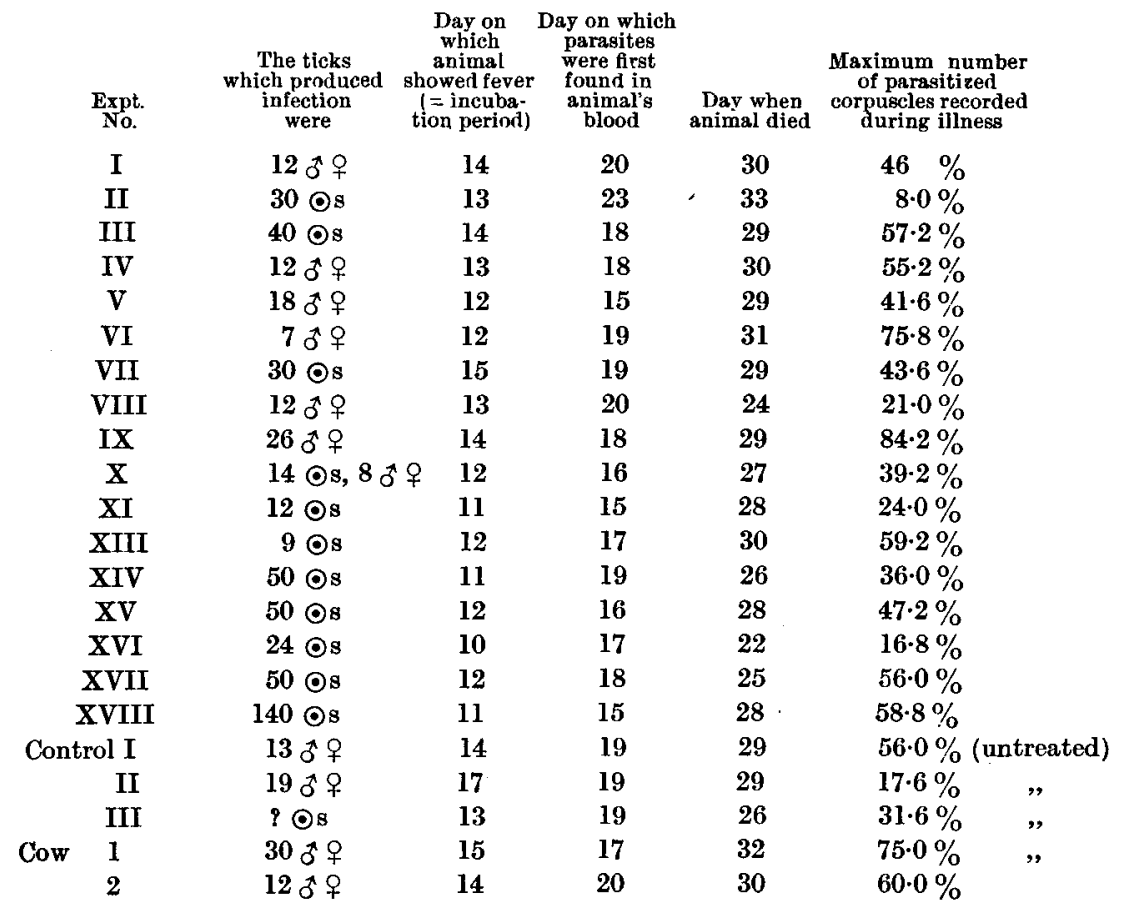

Note: The last two experiments are recorded in Parasitology, II. pp. 328, 329. These animals were infected by means of Rhipicephalus evertsi whereas all of the other animals were infected through our strain of $R$. appendiculatus originally received from Cape Colony through the courtesy of Mr C. P. Lounsbury. 